\title{
Electrowinning of Copper Using Rotating Cylinder Electrode Utilizing Lead Anode
}

\author{
Hesham Soliman ${ }^{1}$, Ahmed Abd El-Moneim ${ }^{2}$ \\ ${ }^{1}$ Advanced Technology and New Materials Research Institute, Mubarak City for Scientific Research and Technology \\ Applications (MuCSAT), Alexandria, Egypt \\ ${ }^{2}$ Materials Science and Engineering Department, Egypt Japan University of Science and Technology, \\ Alexandria, Egypt \\ E-mail:h.soliman@mucsat.sci.eg \\ Received January 10, 2011; revised February 9, 2011; accepted March 7, 2011
}

\begin{abstract}
The effect of lead anode, rotating cylinder electrode (RCE), amount of 1, 2-dihydroxypropane (12-DHP), temperature and rotation on the electrowinning of copper from low concentration acidified copper sulphate solution has been investigated. Copper powder was electrodeposited onto RCE that made of pure copper. From cyclic voltammetry experiments, an empirical parameter called the departure percent, S, was obtained which may represent the stability of the organic additive in the given medium and under the experimental conditions. The inhibition percentage, $\mathrm{P}$, was $0.00-89.91 \%$ depending on the experimental variables. $\mathrm{P}$ was affected by temperature and mole fraction of 12-DHP, while rotation did not show any influence on it. Values of activation energy of electrodeposition process, $E_{a}$, were found to be less than $28 \mathrm{~kJ} \mathrm{~mol}^{-1}$ indicating diffusion controlled process. The overall mass transfer correlations under the present conditions have been computed using the dimensional analysis method. The data were valid for $90<\mathrm{Sh}<1098,737<\mathrm{Sc}<59284$ and $271<\operatorname{Re}<7046$ and the results agreed with the previous studies of mass transfer to rotating cylinders in turbulent flow regimes. The effect of time, content of 12-DHP, temperature and the speed of rotation on the morphological changes of the electrodeposited copper powder as well as deposits composition and crystallite size have been studied. Various crystallite sizes ranged $7.1 \mathrm{~nm}-250.6 \mathrm{~nm}$ were obtained and characterized by EDS and XRD. Different topographs proved that the rate of copper electrodeposition increased by increasing deposition time, temperature and the speed of rotation. Also, they proved that the deposition rate decreased by adding 12-DHP to the solution. Therefore, the results obtained by SEM supported those achieved by measuring the limiting current density and follow the normal manner when organic solvents were added to the electrodeposition bath.
\end{abstract}

Keywords: Electrodeposition, Electrowinning, Lead Anode, Rotating Cylinder Electrode (RCE), 1,2-Dihydroxypropane (12-DHP), Copper Powder

\section{Introduction}

The recovery of metals from aqueous solutions by electrolytic means has been a major factor in extractive technology during the last decades. The source of metal can be an ore as well as waste residue (scrap metals, dusts, sludges collected from metallurgical operations, etc.). One of the aims in studying metal electrowinning is to gain better understanding of the metal electrodeposition process, with emphasis on the role of additives and transport on electro-crystallization. The increase in the world's demand for high-purity copper has sparked an expansion in the production of copper. To produce high-purity copper, copper is sent as a solute in an aqueous solution to the electrowinning plant. In this plant, an electric current is used to remove the copper from solution into flat sheets of metal. These flat sheets are $99.97 \%$ copper, which is the purest form of copper "industrial wise" produced from any production process [1]. It is well known that the introduction of small amounts of certain substances in electrodeposition baths leads to marked changes in the nature of metallic deposits ob- 
tained at the cathode. It is well known that small quantities of these substances may stop dendrites growing and improve significantly the smoothness of the cathodic surface [2]. Thiourea [3], gelatine [4], animal glue [5], polyacrylamides [6] and various mixtures of additives [7] are commonly used as levelling and brightening agents in copper electrodeposition and electrowinning. The development of suitable additives as substitutes for thiourea and gelatine remains an interesting research direction, since it has been established that thiourea decomposes, leading to contamination of the cathodic deposit with sulfur [5]. Within each electrolytic cell, electrical current is passed through the electrolyte from the anode to the cathode. In the copper electrowinning process, the electrolyte is an aqueous solution containing copper sulphate $\left(\mathrm{CuSO}_{4}\right)$ and sulphuric acid $\left(\mathrm{H}_{2} \mathrm{SO}_{4}\right)$ at temperatures ranging from 25 to $45^{\circ} \mathrm{C}$ [1]. As current is passed, copper is removed from the electrolyte and deposited onto the cathode. The potential applied to the cell is terminated once the desired thickness of electrowon copper is reached at the cathode. This takes between 5 and 14 days depending on the applied current density (current per unit area of the electrode). The potential applied to produce the current creates both primary and secondary reactions within the electrolytic cell. At the cathode, a primary reaction deposits copper onto the cathode:

$$
\mathrm{Cu}^{2+}+2 e^{-} \rightarrow \mathrm{Cu}
$$

A secondary reaction, at low concentrations of copper, releases hydrogen bubbles at the cathode:

$$
2 \mathrm{H}^{+}+2 e^{-} \rightarrow \mathrm{H}_{2}
$$

At the anode, a primary reaction occurs that produces oxygen bubbles. The overall reaction occurring at the anode can be seen below:

$$
2 \mathrm{H}_{2} \mathrm{O} \rightarrow \mathrm{O}_{2}+4 \mathrm{H}^{+}+4 e^{-}
$$

Finding the middle ground between mass production in the industry of metal powder extraction and purity as well as size of the produced crystallites is a compromising task, therefore, this work aims to investigate the effect of lead anode and RCE on the electrowinning of copper with respect to the quality and quantity of copper powder; respectively. A parallel goal of this investigation is to study the effect of 12-DHP and temperature as crystallite size, morphology, structure and purity modifiers, which supported by XRD, SEM and EDS.

\section{Experimental}

All chemicals were Analar grade and supplied by BDH chemicals Ltd. Solutions were prepared with water of resistivity $15-\mathrm{M} \Omega \mathrm{cm}$ and copper sulphate concentration and content were checked by the iodine-thiosulphate method [8]. The density and viscosity for all solutions were measured at 298, 303, 308 and $313 \mathrm{~K}$ [9] and the data are listed in Table $\mathbf{1 .}$

A standard $1000 \mathrm{~mL}$ glass-type cell with a polypropylene lid was used in all electrochemical experiments [9]. The polypropylene lid contained four inlets, which were used for working electrode WE immersion, luggin probe (reference electrode RE: saturated calomel elec-

\begin{tabular}{|c|c|c|c|}
\hline & $\mathbf{T}, \mathbf{K}$ & $\eta$, poise & $\rho, \mathbf{g} \cdot \mathrm{cm}^{-3}$ \\
\hline \multirow{4}{*}{ Blank, 0\% (v/v) 12-DHP } & 298 & 0.0107 & 1.0828 \\
\hline & 303 & 0.0096 & 1.0800 \\
\hline & 308 & 0.0087 & 1.0772 \\
\hline & 313 & 0.0079 & 1.0745 \\
\hline \multirow{4}{*}{$10 \%(\mathrm{v} / \mathrm{v}) 12-\mathrm{DHP}$} & 298 & 0.0112 & 1.0900 \\
\hline & 303 & 0.0100 & 1.0872 \\
\hline & 308 & 0.0091 & 1.0844 \\
\hline & 313 & 0.0082 & 1.0816 \\
\hline \multirow{4}{*}{$20 \%$ (v/v) 12-DHP } & 298 & 0.0129 & 1.0981 \\
\hline & 303 & 0.0116 & 1.0953 \\
\hline & 308 & 0.0105 & 1.0925 \\
\hline & 313 & 0.0095 & 1.0897 \\
\hline \multirow{4}{*}{$30 \%(\mathrm{v} / \mathrm{v}) 12-\mathrm{DHP}$} & 298 & 0.0153 & 1.1112 \\
\hline & 303 & 0.0137 & 1.1083 \\
\hline & 308 & 0.0124 & 1.1055 \\
\hline & 313 & 0.0113 & 1.1027 \\
\hline \multirow{4}{*}{$40 \%(\mathrm{v} / \mathrm{v}) 12-\mathrm{DHP}$} & 298 & 0.0186 & 1.1193 \\
\hline & 303 & 0.0167 & 1.1165 \\
\hline & 308 & 0.0151 & 1.1136 \\
\hline & 313 & 0.0137 & 1.1107 \\
\hline \multirow{4}{*}{$50 \%(\mathrm{v} / \mathrm{v}) 12-\mathrm{DHP}$} & 298 & 0.0231 & 1.1314 \\
\hline & 303 & 0.0207 & 1.1285 \\
\hline & 308 & 0.0187 & 1.1256 \\
\hline & 313 & 0.0170 & 1.1227 \\
\hline \multirow{4}{*}{$60 \%$ (v/v) 12-DHP } & 298 & 0.0293 & 1.1406 \\
\hline & 303 & 0.0263 & 1.1376 \\
\hline & 308 & 0.0238 & 1.1347 \\
\hline & 313 & 0.0216 & 1.1318 \\
\hline
\end{tabular}

Table 1. Values of Viscosity, $\eta$, and density, $\rho$, for all solutions at different temperatures. 
trode, SCE), counter electrode CE (lead sheet) and thermometer to measure the temperature. A $99.9 \%$ purity copper cylindrical sample was used as WE. This sample was rotated at controlled rates by an analytical rotator model A.S.R. made by PINE Instrument Company. The cylindrical samples of copper had dimensions of $8 \mathrm{~mm}$ height and $12 \mathrm{~mm}$ diameter. The specimens were lightly abraded with 1200 grade silicon carbide paper, washed with distilled water, acetone and deionized water and dried in a cool air stream prior to use. The anode CE, was machined from analytical highly pure lead sheet (99.99\%) with exposed surface area of $5 \mathrm{~cm} \times 10 \mathrm{~cm}$. the separation distance between WE and CE was kept constant at $5 \mathrm{~cm}$ for all experiments. In order to ensure a correct control of the specimen rotation rate and the correct recording of potentials, regular checking of the rotation rate was carried out.

The Potentiostat was Ministat Precision Potentiostat made by Thompson Electrochem Ltd. coupled to a Chemical Electronics (Birtley) Ltd. Sweep Generator and controlled by a PC via an Advantech PCL-718 Lab Card and a PCLD-780 wiring terminal board. In this system the Advantech Labtech Aquire software was used. All electrochemical experiments and polarization curves were carried out using a potentiodynamic method. The sweep rate in all experiments was $100 \mathrm{mV} \cdot \mathrm{min}^{-1}$, which considered being suitable for copper electrodeposition [10].

\subsection{Cathodic Polarization}

The variables used in this investigation were rotation speed $(0-1000 \mathrm{rpm})$, temperature $(298-313 \mathrm{~K})$, and blank solution $\left(0.1 \mathrm{~mol} \cdot \mathrm{L}^{-1} \mathrm{CuSO}_{4}\right.$ and $1.5 \mathrm{~mol} \cdot \mathrm{L}^{-1}$ $\left.\mathrm{H}_{2} \mathrm{SO}_{4}\right)$ with and without 12-DHP $10-60 \%(\mathrm{v} / \mathrm{v})$ with a sweep range of $700 \mathrm{mV}$ starting from the open circuit potential.

\subsection{Cyclic Voltammetry}

The main purpose of this part is to make sure that the solution under investigation does not change by changing the temperature or the applied potential. The polarization range in these experiments was +0.1 to $-0.9 \mathrm{~V}$ vs. SCE. The polarization was potentiodynamic without rotation, i.e. free convection with employing lead as anode. The variables, were temperature $(298-313 \mathrm{~K})$ and blank solution $\left(0.1 \mathrm{~mol} \cdot \mathrm{L}^{-1} \mathrm{CuSO}_{4}\right.$ and $\left.1.5 \mathrm{~mol} \cdot \mathrm{L}^{-1} \mathrm{H}_{2} \mathrm{SO}_{4}\right)$ with and without 12-DHP 10\%-60\% (v/v). The polarization was cyclic polarization begins at $+0.1 \mathrm{~V}$ and ends the half cycle at $-0.9 \mathrm{~V}$, then the second half cycle begins at $-0.9 \mathrm{~V}$ and ends the whole cycle at $+0.1 \mathrm{~V}$ where the experiment started.

\subsection{Morphology and Composition Analyses}

An AMRAY 1810 Scanning Electron Microscope (SEM) was used to examine the surface topography of specimens after experiment, while Energy Dispersive X-Ray Analysis (EDS) was used for elemental analyses. The specimens were examined through an acceleration potential of $20 \mathrm{KeV}$; the specimens were coated with carbon to avoid electron charging of the surface. The specimens were made of copper rods that were cut into small pieces of dimensions $0.8 \mathrm{~cm}$ height and $1.2 \mathrm{~cm}$ diameter. The surfaces for SEM analyses were polished with series of silicon carbide paper grades $120 \mu \mathrm{m}-1200 \mu \mathrm{m}$, then with $3 \mu \mathrm{m}$ diamond paste followed by $1 \mu \mathrm{m}$ diamond paste on a polishing wheel. After washing with deionized water and degreasing in acetone, warm air was blown over the surface. These specimens were stored in a desiccator over silica gel prior to use. Temperature; $298 \mathrm{~K}$ - $313 \mathrm{~K}, 12-\mathrm{DHP}$ content; 0 - 60\% (v/v), rotation rate; 0 - 1000 rpm; and time; 10 - 30 minutes; were the different variables that have been employed to study the surface topography by SEM. X-ray diffraction (XRD) measurement for copper powder was carried out using a Shimazu XRD-7000 X-Ray Diffractometer with $\mathrm{Cu}-\mathrm{K}_{1} \alpha_{1}$ radiation operated at $40 \mathrm{kV}$ and $30 \mathrm{~mA}$, with a scan speed of $2 \theta$ $\min ^{-1}$.

\section{Results and Discussion}

\subsection{Cyclic Voltammetry}

The stability of organic additives, whether they are solids or liquids, plays an essential role to maintain the bath reproducibility and in turn affects the deposit quality. In order to check the stability of the present bath, cyclic voltammetry experiments were carried out for all solutions at different temperatures in the natural convection regime. This cyclic voltammetry led to compare the values of the limiting current density in the first half cycle $\left(i_{l-1 s t}\right)$ with those in the second $\left(i_{l-2 n d}\right)$ and an empirical parameter, S, was obtained using Equation (4), where $S$ is called the departure percent:

$$
S=\frac{i_{l_{1 s t}}}{i_{l_{2 n d}}} \times 100
$$

Table 2 summarizes the values of $\mathrm{S}$ for all solutions at all experimental conditions, while Figure 1 represents the variation of $\mathrm{S}$ with the mole fraction of 12-DHP at $298 \mathrm{~K}$ and $313 \mathrm{~K}$ in the natural convection regime. Figure 1 shows that $S$ decreases with increasing temperature in all proportions of 12-DHP; while within the same temperature range of $298 \mathrm{~K}$ it increases up to $30 \% 12$-DHP $(\mathrm{v} / \mathrm{v})$ then fluctuates downwards between $40 \%$ and $50 \%$ 


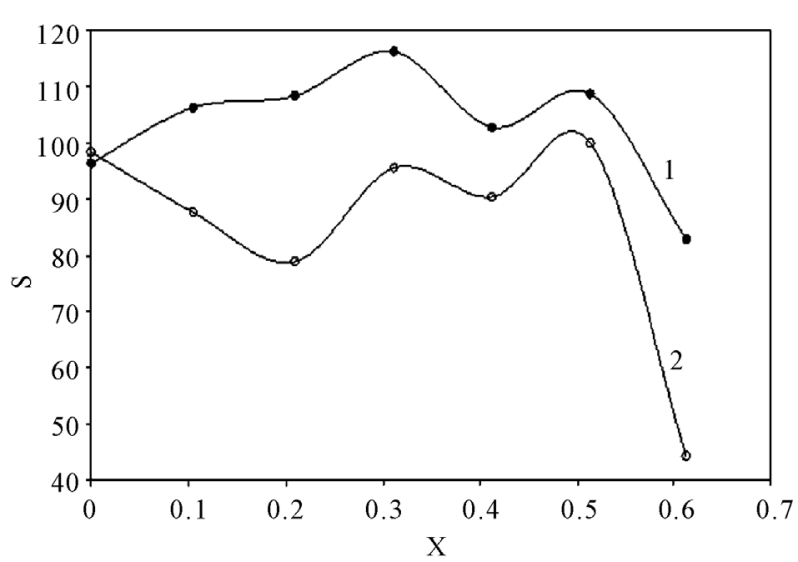

Figure 1. Variation of the departure percent, $S$, with the mole fraction, $X$, of 12-DHP at zero rotation and at (1) 298 K, (2) $313 \mathrm{~K}$.

Table 2. Values of the average departure percent, $\mathrm{S}$, for all solutions at different temperatures.

\begin{tabular}{ccccc}
\hline & \multicolumn{4}{c}{$S$} \\
\cline { 2 - 5 } & At 298 K & At 303 K & At 308 K & At 313 K \\
\hline Blank, 0\% (v/v) 12-DHP & 96.4 & 97.4 & 96.3 & 98.4 \\
$10 \%(\mathrm{v} / \mathrm{v})$ 12-DHP & 106.2 & 99.5 & 105.1 & 87.7 \\
20\% (v/v) 12-DHP & 108.3 & 97.6 & 91.5 & 78.9 \\
$30 \%(\mathrm{v} / \mathrm{v}) 12-\mathrm{DHP}$ & 116.1 & 120.7 & 90.8 & 95.6 \\
40\% (v/v) 12-DHP & 102.7 & 102.7 & 102.6 & 90.4 \\
$50 \%(\mathrm{v} / \mathrm{v})$ 12-DHP & 108.7 & 90.8 & 83.0 & 100.0 \\
$60 \%$ (v/v) 12-DHP & 82.9 & 48.4 & 44.3 & 44.3 \\
\hline
\end{tabular}

12-DHP $(\mathrm{v} / \mathrm{v})$ and finally reaches the minimum value at $60 \% 12-\mathrm{DHP}(\mathrm{v} / \mathrm{v})$. At $313 \mathrm{~K}$ it decreases up to $20 \%$ 12-DHP (v/v) then fluctuates upwards between 30 and $50 \% 12-\mathrm{DHP}(\mathrm{v} / \mathrm{v})$ and lasts with the minimum value at $60 \%$ 12-DHP (v/v). As an explanation of Figure 1, the departure percent, S, may represent the stability of the organic additive in the given medium and under the effect of the experimental conditions, more investigation is needed to generalize this definition. Nevertheless, if controlling the sweep rate could reduce this departure, then it will consider as a simple, cheap and precise method of estimating the right sweep rate that is valuable in industrial electrochemistry. In addition, it considered as an early warning test in case of using organic solvents, since organic solvents are easily evaporate and decompose.

\subsection{Cathodic Polarisation}

Values of the limiting current densities were measured from the polarization curves of all solutions at different rotations and different temperatures are given in Table 3. It is clear in Table 3 that the limiting current density increases with increasing the speed of rotation, which proves that the electrodeposition (electrowinning) process of copper in presence of 12-DHP as well as in aqueous media is diffusion-controlled reaction [2]. The relation between the limiting current densities, $i_{1}$, and the mole fractions, X, for 12-DHP solutions at different temperatures and $500 \mathrm{rpm}$ is revealed in Figure 2 as an example. Noticeably, the limiting current density decreases with increasing 12-DHP mole fraction and increases by increasing temperature.

The increase of mass transport under forced or turbulent conditions may refer to [11] solution temperature elevation, roughness of the deposit and/or the increase of agitation. Equation (5) was used to calculate the mass transfer coefficients, $\mathrm{k}$, for 12-DHP solutions.

$$
k=\frac{i_{l}}{n F C_{\text {。 }}}
$$

where: $n=$ number of electrons involved in the reaction, $F=$ Faraday constant $=96500\left(\right.$ coulomb $\mathrm{mol}^{-1}$, coulomb $=$ A.s.), $\mathrm{C}_{\mathrm{o}}$ bulk concentration of copper sulphate (mol $\mathrm{cm}^{-3}$ ).

The percentage of inhibition efficiency, $\mathrm{P}$, was calculated from Equation (6) and its values are listed in Table 4. The percentage of inhibition caused by 12 -DHP was in the range of $0.00-89.91 \%$ depending on the concentration of 12-DHP and the temperature. The relation between the percentage of inhibition, $\mathrm{P}$, and the mole fraction of 12-DHP, $\mathrm{X}$, at $308 \mathrm{~K}$ and different rotations is represented in Figure $\mathbf{3}$ as an example. It is observed from Figure 3 and Table 4 that $P$ increased as $X$ increased and as temperature decreased, but it was not affected by rotation. This indicates that the rotation factor

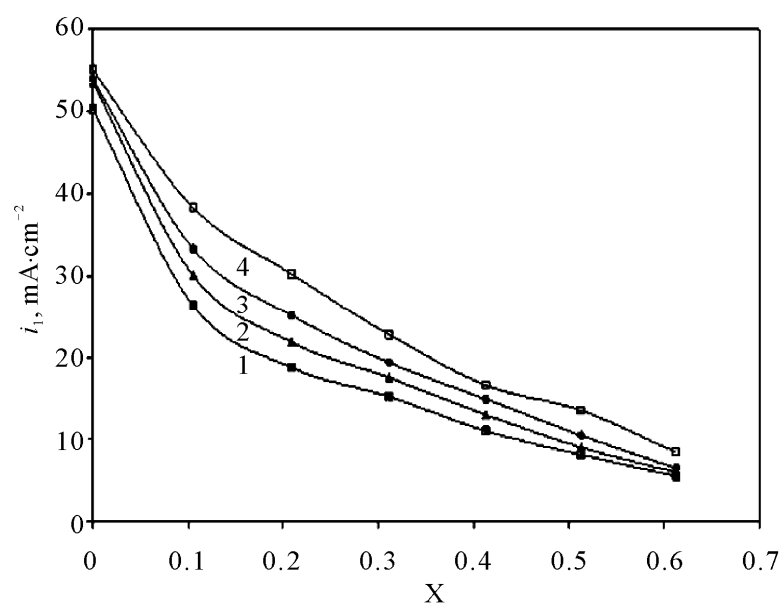

Figure 2. Variation of limiting current density, $i_{\mathrm{b}}$, with the mole fraction, $X$, of 12-DHP at $500 \mathrm{rpm}$ and at (1) $298 \mathrm{~K}$, (2) $303 \mathrm{~K}$, (3) $308 \mathrm{~K}$, (4) $313 \mathrm{~K}$. 
Table 3. Values of the limiting current density, $i_{l}$, for all solutions at different temperatures and different speeds of rotation.

\begin{tabular}{|c|c|c|c|c|c|}
\hline & \multirow{2}{*}{ rpm } & \multicolumn{4}{|c|}{$i_{\mathrm{l}}, \mathrm{mA} \cdot \mathrm{cm}^{-2}$} \\
\hline & & $298 \mathrm{~K}$ & $303 \mathrm{~K}$ & $308 \mathrm{~K}$ & $313 \mathrm{~K}$ \\
\hline \multirow{16}{*}{ Blank, 0\% (v/v) 12-DHP } & 0 & 8.44 & 8.96 & 9.39 & 9.45 \\
\hline & 100 & 20.24 & 21.59 & 22.27 & 22.95 \\
\hline & 200 & 29.82 & 32.36 & 33.11 & 33.88 \\
\hline & 300 & 37.32 & 40.90 & 41.05 & 42.43 \\
\hline & 400 & 46.49 & 47.84 & 48.20 & 48.55 \\
\hline & 500 & 50.37 & 53.56 & 53.98 & 55.06 \\
\hline & 600 & 55.03 & 57.67 & 58.83 & 59.97 \\
\hline & 700 & 62.23 & 63.45 & 63.90 & 64.33 \\
\hline & 800 & 66.65 & 67.36 & 67.43 & 67.51 \\
\hline & 900 & 72.07 & 72.82 & 73.55 & 74.27 \\
\hline & 1000 & 77.71 & 77.97 & 78.11 & 78.25 \\
\hline & 0 & 6.48 & 6.75 & 6.92 & 8.23 \\
\hline & 100 & 10.09 & 11.57 & 12.13 & 15.64 \\
\hline & 200 & 15.47 & 16.81 & 19.88 & 22.05 \\
\hline & 300 & 19.26 & 22.32 & 24.90 & 27.73 \\
\hline & 400 & 23.15 & 26.52 & 29.14 & 33.34 \\
\hline \multirow[t]{11}{*}{$10 \%(\mathrm{v} / \mathrm{v}) 12-\mathrm{DHP}$} & 500 & 26.35 & 30.04 & 33.28 & 38.30 \\
\hline & 600 & 29.93 & 33.48 & 37.27 & 43.13 \\
\hline & 700 & 32.86 & 36.82 & 41.40 & 48.05 \\
\hline & 800 & 35.14 & 39.72 & 44.40 & 54.36 \\
\hline & 900 & 37.55 & 41.09 & 47.50 & 59.66 \\
\hline & 1000 & 40.23 & 45.06 & 50.19 & 59.87 \\
\hline & 0 & 4.71 & 5.08 & 5.28 & 5.95 \\
\hline & 100 & 6.49 & 9.63 & 10.20 & 11.57 \\
\hline & 200 & 11.40 & 12.74 & 15.45 & 17.22 \\
\hline & 300 & 14.34 & 16.12 & 19.16 & 21.97 \\
\hline & 400 & 16.35 & 19.06 & 22.00 & 26.62 \\
\hline \multirow[t]{7}{*}{$20 \%(\mathrm{v} / \mathrm{v}) 12-\mathrm{DHP}$} & 500 & 18.76 & 21.87 & 25.14 & 30.26 \\
\hline & 600 & 21.53 & 24.51 & 28.45 & 32.63 \\
\hline & 700 & 23.24 & 26.21 & 31.46 & 39.49 \\
\hline & 800 & 25.14 & 28.59 & 33.30 & 40.86 \\
\hline & 900 & 26.68 & 30.73 & 36.14 & 49.52 \\
\hline & 1000 & 28.59 & 33.07 & 40.22 & 53.06 \\
\hline & 0 & 3.68 & 3.75 & 3.93 & 4.49 \\
\hline \multirow{3}{*}{$30 \%(\mathrm{v} / \mathrm{v}) 12-\mathrm{DHP}$} & 100 & 6.69 & 7.60 & 8.17 & 9.18 \\
\hline & 200 & 9.01 & 10.30 & 11.11 & 13.56 \\
\hline & 300 & 11.49 & 12.90 & 15.38 & 16.89 \\
\hline
\end{tabular}




\begin{tabular}{|c|c|c|c|c|c|}
\hline & 400 & 13.07 & 15.14 & 17.14 & 19.62 \\
\hline & 500 & 15.21 & 17.52 & 19.34 & 22.78 \\
\hline & 600 & 16.75 & 19.55 & 21.62 & 25.51 \\
\hline & 700 & 18.68 & 21.38 & 23.58 & 27.89 \\
\hline & 800 & 19.87 & 22.60 & 24.29 & 29.54 \\
\hline & 900 & 20.96 & 23.72 & 27.51 & 32.03 \\
\hline & 1000 & 22.53 & 25.72 & 29.30 & 33.29 \\
\hline & 0 & 2.52 & 2.70 & 2.84 & 3.15 \\
\hline & 100 & 4.66 & 6.13 & 6.27 & 6.69 \\
\hline & 200 & 7.39 & 8.66 & 9.95 & 10.13 \\
\hline & 300 & 8.69 & 9.60 & 10.93 & 12.27 \\
\hline & 400 & 10.44 & 11.21 & 12.62 & 14.51 \\
\hline \multirow[t]{11}{*}{$40 \%(\mathrm{v} / \mathrm{v}) 12-\mathrm{DHP}$} & 500 & 11.04 & 13.00 & 14.89 & 16.61 \\
\hline & 600 & 12.93 & 14.09 & 16.51 & 19.27 \\
\hline & 700 & 13.70 & 15.24 & 17.63 & 19.52 \\
\hline & 800 & 14.33 & 17.49 & 20.26 & 21.13 \\
\hline & 900 & 15.35 & 17.73 & 21.06 & 24.29 \\
\hline & 1000 & 16.16 & 18.78 & 22.92 & 24.85 \\
\hline & 0 & 1.58 & 1.75 & 1.81 & 1.99 \\
\hline & 100 & 3.75 & 4.24 & 4.69 & 5.02 \\
\hline & 200 & 5.40 & 5.99 & 7.33 & 8.19 \\
\hline & 300 & 6.97 & 7.60 & 8.99 & 9.75 \\
\hline & 400 & 7.71 & 8.97 & 9.78 & 10.83 \\
\hline \multirow[t]{11}{*}{$50 \%(\mathrm{v} / \mathrm{v}) 12-\mathrm{DHP}$} & 500 & 8.17 & 9.08 & 10.50 & 13.53 \\
\hline & 600 & 8.38 & 9.88 & 11.77 & 14.98 \\
\hline & 700 & 9.46 & 11.35 & 13.07 & 16.31 \\
\hline & 800 & 10.37 & 11.42 & 14.11 & 17.47 \\
\hline & 900 & 10.65 & 13.39 & 15.48 & 18.30 \\
\hline & 1000 & 11.60 & 14.58 & 16.42 & 18.95 \\
\hline & 0 & 1.13 & 1.23 & 1.26 & 1.37 \\
\hline & 100 & 2.35 & 2.53 & 2.56 & 2.63 \\
\hline & 200 & 3.28 & 3.34 & 3.72 & 4.61 \\
\hline & 300 & 4.03 & 4.73 & 5.12 & 5.43 \\
\hline & 400 & 5.05 & 5.05 & 6.48 & 7.44 \\
\hline \multirow[t]{6}{*}{$60 \%(\mathrm{v} / \mathrm{v}) 12-\mathrm{DHP}$} & 500 & 5.56 & 6.03 & 6.55 & 8.50 \\
\hline & 600 & 6.21 & 6.31 & 8.19 & 9.59 \\
\hline & 700 & 6.28 & 7.75 & 9.49 & 10.99 \\
\hline & 800 & 7.44 & 9.08 & 11.26 & 12.28 \\
\hline & 900 & 7.44 & 9.76 & 12.39 & 12.49 \\
\hline & 1000 & 8.12 & 10.78 & 12.90 & 13.51 \\
\hline
\end{tabular}


Table 4. Values of inhibition percent, $P$, for 12-DHP solutions at different temperatures with different speeds of rotation.

\begin{tabular}{|c|c|c|c|c|c|}
\hline & \multirow{2}{*}{ rpm } & \multicolumn{4}{|c|}{$P$} \\
\hline & & $298 \mathrm{~K}$ & $303 \mathrm{~K}$ & $308 \mathrm{~K}$ & $313 \mathrm{~K}$ \\
\hline Blank, 0\% (v/v) 12-DHP & & 0.00 & 0.00 & 0.00 & 0.00 \\
\hline $10 \%(\mathrm{v} / \mathrm{v}) 12-\mathrm{DHP}$ & & 23.27 & 24.68 & 26.26 & 12.89 \\
\hline $20 \%(\mathrm{v} / \mathrm{v}) 12-\mathrm{DHP}$ & & 44.15 & 43.30 & 43.74 & 37.02 \\
\hline $30 \%(\mathrm{v} / \mathrm{v}) 12-\mathrm{DHP}$ & 0 & 56.40 & 58.16 & 58.20 & 52.53 \\
\hline $40 \%(\mathrm{v} / \mathrm{v}) 12-\mathrm{DHP}$ & & 70.11 & 69.90 & 69.78 & 66.63 \\
\hline $50 \%(\mathrm{v} / \mathrm{v}) 12-\mathrm{DHP}$ & & 81.32 & 80.45 & 80.78 & 79.00 \\
\hline $60 \%(\mathrm{v} / \mathrm{v}) 12-\mathrm{DHP}$ & & 86.66 & 86.31 & 86.56 & 85.56 \\
\hline Blank, $0 \%(\mathrm{v} / \mathrm{v}) 12-\mathrm{DHP}$ & & 0.00 & 0.00 & 0.00 & 0.00 \\
\hline $10 \%(\mathrm{v} / \mathrm{v}) 12-\mathrm{DHP}$ & & 50.14 & 46.39 & 45.55 & 31.86 \\
\hline $20 \%(\mathrm{v} / \mathrm{v}) 12-\mathrm{DHP}$ & & 67.96 & 55.40 & 54.21 & 49.59 \\
\hline $30 \%(v / v) 12-D H P$ & 100 & 66.94 & 64.78 & 63.34 & 60.00 \\
\hline $40 \%(\mathrm{v} / \mathrm{v}) 12-\mathrm{DHP}$ & & 76.98 & 71.59 & 71.83 & 70.84 \\
\hline $50 \%(\mathrm{v} / \mathrm{v}) 12-\mathrm{DHP}$ & & 81.48 & 80.36 & 78.93 & 78.14 \\
\hline $60 \%(\mathrm{v} / \mathrm{v}) 12-\mathrm{DHP}$ & & 88.37 & 88.30 & 88.51 & 88.55 \\
\hline Blank, 0\% (v/v) 12-DHP & & 0.00 & 0.00 & 0.00 & 0.00 \\
\hline $10 \%(\mathrm{v} / \mathrm{v}) 12-\mathrm{DHP}$ & & 48.13 & 48.05 & 39.97 & 34.93 \\
\hline $20 \%(\mathrm{v} / \mathrm{v}) 12-\mathrm{DHP}$ & & 61.76 & 60.63 & 53.35 & 49.18 \\
\hline $30 \%(v / v) 12-D H P$ & 200 & 69.80 & 68.16 & 66.45 & 59.97 \\
\hline $40 \%(\mathrm{v} / \mathrm{v}) 12-\mathrm{DHP}$ & & 75.20 & 73.25 & 69.94 & 70.11 \\
\hline $50 \%(\mathrm{v} / \mathrm{v}) 12-\mathrm{DHP}$ & & 81.90 & 81.48 & 77.87 & 75.82 \\
\hline $60 \%(\mathrm{v} / \mathrm{v}) 12-\mathrm{DHP}$ & & 89.02 & 89.67 & 88.77 & 86.40 \\
\hline Blank, 0\% (v/v) 12-DHP & & 0.00 & 0.00 & 0.00 & 0.00 \\
\hline $10 \%(\mathrm{v} / \mathrm{v}) 12-\mathrm{DHP}$ & & 48.40 & 45.43 & 39.33 & 34.65 \\
\hline $20 \%(\mathrm{v} / \mathrm{v}) 12-\mathrm{DHP}$ & & 61.56 & 60.60 & 53.33 & 48.23 \\
\hline $30 \%(\mathrm{v} / \mathrm{v}) 12-\mathrm{DHP}$ & 300 & 69.20 & 68.47 & 62.52 & 60.19 \\
\hline $40 \%(\mathrm{v} / \mathrm{v}) 12-\mathrm{DHP}$ & & 76.71 & 76.52 & 73.37 & 71.09 \\
\hline $50 \%(\mathrm{v} / \mathrm{v}) 12-\mathrm{DHP}$ & & 81.31 & 81.41 & 78.11 & 77.03 \\
\hline $60 \%(\mathrm{v} / \mathrm{v}) 12-\mathrm{DHP}$ & & 89.21 & 88.44 & 87.53 & 87.21 \\
\hline Blank, 0\% (v/v) 12-DHP & & 0.00 & 0.00 & 0.00 & 0.00 \\
\hline $10 \%(\mathrm{v} / \mathrm{v}) 12-\mathrm{DHP}$ & & 50.21 & 44.56 & 39.54 & 31.32 \\
\hline $20 \%(\mathrm{v} / \mathrm{v}) 12-\mathrm{DHP}$ & & 64.83 & 60.16 & 54.35 & 45.18 \\
\hline $30 \%(\mathrm{v} / \mathrm{v}) 12-\mathrm{DHP}$ & 400 & 71.89 & 68.35 & 64.45 & 59.58 \\
\hline $40 \%(\mathrm{v} / \mathrm{v}) 12-\mathrm{DHP}$ & & 77.54 & 76.56 & 73.83 & 70.12 \\
\hline $50 \%(\mathrm{v} / \mathrm{v}) 12-\mathrm{DHP}$ & & 83.42 & 81.25 & 79.71 & 77.70 \\
\hline $60 \%(\mathrm{v} / \mathrm{v}) 12-\mathrm{DHP}$ & & 89.14 & 89.44 & 86.55 & 84.68 \\
\hline Blank, $0 \%(\mathrm{v} / \mathrm{v}) 12-\mathrm{DHP}$ & 5 & 0.00 & 0.00 & 0.00 & 0.00 \\
\hline $10 \%(\mathrm{v} / \mathrm{v}) 12-\mathrm{DHP}$ & 700 & 47.68 & 43.92 & 38.36 & 30.43 \\
\hline
\end{tabular}




\begin{tabular}{|c|c|c|c|c|c|}
\hline $20 \%(\mathrm{v} / \mathrm{v}) 12-\mathrm{DHP}$ & & 62.76 & 59.17 & 53.42 & 45.04 \\
\hline $30 \%(\mathrm{v} / \mathrm{v}) 12-\mathrm{DHP}$ & & 69.80 & 67.29 & 64.16 & 58.63 \\
\hline $40 \%(\mathrm{v} / \mathrm{v}) 12-\mathrm{DHP}$ & & 78.08 & 75.73 & 72.41 & 69.83 \\
\hline $50 \%(\mathrm{v} / \mathrm{v}) 12-\mathrm{DHP}$ & & 83.79 & 83.05 & 80.54 & 75.42 \\
\hline $60 \%(\mathrm{v} / \mathrm{v}) 12-\mathrm{DHP}$ & & 88.96 & 88.75 & 87.86 & 84.57 \\
\hline Blank, $0 \%$ (v/v) 12-DHP & & 0.00 & 0.00 & 0.00 & 0.00 \\
\hline $10 \%(\mathrm{v} / \mathrm{v}) 12-\mathrm{DHP}$ & & 45.60 & 41.94 & 36.65 & 28.09 \\
\hline $20 \%(\mathrm{v} / \mathrm{v}) 12-\mathrm{DHP}$ & & 60.87 & 57.50 & 51.63 & 45.58 \\
\hline $30 \%(\mathrm{v} / \mathrm{v}) 12-\mathrm{DHP}$ & 600 & 69.56 & 66.09 & 63.25 & 57.46 \\
\hline $40 \%(\mathrm{v} / \mathrm{v}) 12-\mathrm{DHP}$ & & 76.50 & 75.57 & 71.94 & 67.86 \\
\hline $50 \%(\mathrm{v} / \mathrm{v}) 12-\mathrm{DHP}$ & & 84.78 & 82.86 & 80.00 & 75.02 \\
\hline $60 \%(\mathrm{v} / \mathrm{v}) 12-\mathrm{DHP}$ & & 88.72 & 89.05 & 86.08 & 84.01 \\
\hline Blank, $0 \%$ (v/v) 12-DHP & & 0.00 & 0.00 & 0.00 & 0.00 \\
\hline $10 \%(\mathrm{v} / \mathrm{v}) 12-\mathrm{DHP}$ & & 47.19 & 41.97 & 35.21 & 25.30 \\
\hline $20 \%(\mathrm{v} / \mathrm{v}) 12-\mathrm{DHP}$ & & 62.66 & 58.69 & 50.76 & 38.62 \\
\hline $30 \%(\mathrm{v} / \mathrm{v}) 12-\mathrm{DHP}$ & 700 & 69.98 & 66.31 & 63.09 & 56.64 \\
\hline $40 \%(\mathrm{v} / \mathrm{v}) 12-\mathrm{DHP}$ & & 77.98 & 75.97 & 72.41 & 69.66 \\
\hline $50 \%(\mathrm{v} / \mathrm{v}) 12-\mathrm{DHP}$ & & 84.79 & 82.11 & 79.55 & 74.64 \\
\hline $60 \%(\mathrm{v} / \mathrm{v}) 12-\mathrm{DHP}$ & & 89.91 & 87.79 & 85.16 & 82.92 \\
\hline Blank, $0 \%$ (v/v) 12-DHP & & 0.00 & 0.00 & 0.00 & 0.00 \\
\hline $10 \%(\mathrm{v} / \mathrm{v}) 12-\mathrm{DHP}$ & & 47.28 & 41.04 & 34.15 & 19.48 \\
\hline $20 \%(\mathrm{v} / \mathrm{v}) 12-\mathrm{DHP}$ & & 62.27 & 57.56 & 50.61 & 39.48 \\
\hline $30 \%(\mathrm{v} / \mathrm{v}) 12-\mathrm{DHP}$ & 800 & 70.19 & 66.44 & 63.98 & 56.24 \\
\hline $40 \%(\mathrm{v} / \mathrm{v}) 12-\mathrm{DHP}$ & & 78.49 & 74.04 & 69.96 & 68.70 \\
\hline $50 \%(\mathrm{v} / \mathrm{v}) 12-\mathrm{DHP}$ & & 84.44 & 83.04 & 79.07 & 74.13 \\
\hline $60 \%(\mathrm{v} / \mathrm{v}) 12-\mathrm{DHP}$ & & 88.84 & 86.53 & 83.30 & 81.81 \\
\hline Blank, 0\% (v/v) 12-DHP & & 0.00 & 0.00 & 0.00 & 0.00 \\
\hline $10 \%(\mathrm{v} / \mathrm{v}) 12-\mathrm{DHP}$ & & 47.91 & 43.57 & 35.42 & 19.67 \\
\hline $20 \%(\mathrm{v} / \mathrm{v}) 12-\mathrm{DHP}$ & & 62.98 & 57.80 & 50.86 & 33.32 \\
\hline $30 \%(\mathrm{v} / \mathrm{v}) 12-\mathrm{DHP}$ & 900 & 70.92 & 67.42 & 62.60 & 56.87 \\
\hline $40 \%(\mathrm{v} / \mathrm{v}) 12-\mathrm{DHP}$ & & 78.70 & 75.65 & 71.37 & 67.30 \\
\hline $50 \%(\mathrm{v} / \mathrm{v}) 12-\mathrm{DHP}$ & & 85.22 & 81.62 & 78.95 & 75.36 \\
\hline $60 \%(\mathrm{v} / \mathrm{v}) 12-\mathrm{DHP}$ & & 89.68 & 86.60 & 83.16 & 83.19 \\
\hline Blank, 0\% (v/v) 12-DHP & & 0.00 & 0.00 & 0.00 & 0.00 \\
\hline $10 \%(\mathrm{v} / \mathrm{v}) 12-\mathrm{DHP}$ & & 48.23 & 42.21 & 35.75 & 23.49 \\
\hline $20 \%(\mathrm{v} / \mathrm{v}) 12-\mathrm{DHP}$ & & 63.21 & 57.59 & 48.50 & 32.19 \\
\hline $30 \%(\mathrm{v} / \mathrm{v}) 12-\mathrm{DHP}$ & 1000 & 71.00 & 67.01 & 62.49 & 57.46 \\
\hline $40 \%(\mathrm{v} / \mathrm{v}) 12-\mathrm{DHP}$ & & 79.21 & 75.91 & 70.66 & 68.25 \\
\hline $50 \%(\mathrm{v} / \mathrm{v}) 12-\mathrm{DHP}$ & & 85.07 & 81.30 & 78.98 & 75.79 \\
\hline $60 \%(\mathrm{v} / \mathrm{v}) 12-\mathrm{DHP}$ & & 89.55 & 86.17 & 83.49 & 82.73 \\
\hline
\end{tabular}




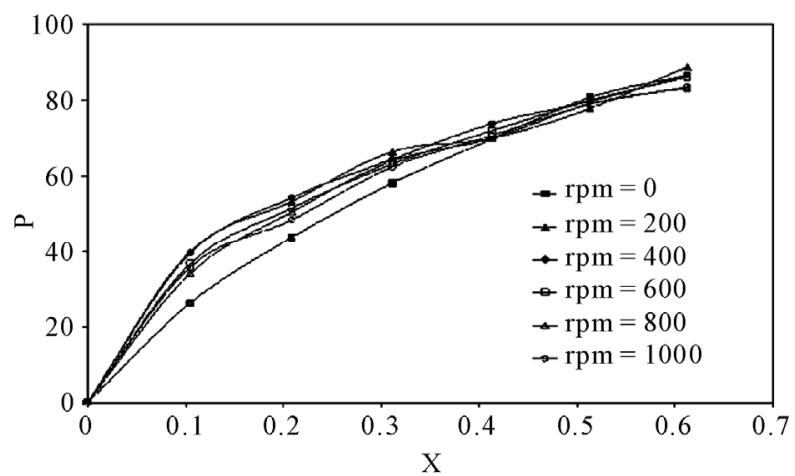

Figure 3. Variation of the inhibition percentage, $P$, with the mole fraction, $\mathrm{X}$, of $12-\mathrm{DHP}$ at $308 \mathrm{~K}$ with different speeds of rotation.

has little effect on the adsorption process and the decrease in mass transfer coefficient in this case is attributable to the increase in the interfacial viscosity [12], which caused by the adsorption of alcohol molecules at the cylinder surface with their non-polar ends, while the polar ones are directed towards solution.

$$
P=\frac{i_{l_{\text {blank }}}-i_{\text {lalc. }}}{i_{\text {blank }}} \times 100
$$

To investigate the mechanism of electrodeposition process, the activation energies, $E_{a}$, of the process were calculated from the values of the limiting current densities in presence and in absence of 12-DHP at different temperatures according to Arrhenius equation:

$$
\ln \left(i_{l}\right)=\ln (A)-\frac{E_{a}}{R} \frac{1}{T}
$$

where $A$ is a pre-exponential factor related to concentration, steric effects, metal surface characteristics...etc; $R$ is the molar gas constant and $T$ is the absolute temperature.

Figure 4 shows the relation between $\ln \left(\mathrm{i}_{1}\right)$ against $1 / \mathrm{T}$ for the 12-DHP solutions at $500 \mathrm{rpm}$ as an example. Straight lines were obtained and $\mathrm{E}_{\mathrm{a}}$ values were calculated using Equation (7) and their values are given in Table 5. Thermodynamic parameters, the enthalpy of activation, $\Delta \mathrm{H}^{*}$, entropy of activation, $\Delta \mathrm{S}^{*}$, and free energy of activation, $\Delta \mathrm{G}^{*}$, were calculated and their values are also listed in Table 5.

The activation energy of the process, $E_{a}$, is an important parameter for determining the rate-controlling step. If the rate-controlling step [13] is the diffusion of aqueous species in the boundary layer then $\mathrm{E}_{\mathrm{a}}$ is generally $\mathrm{O} \leq$ $28 \mathrm{~kJ} \mathrm{~mol}^{-1}$, while $E_{a}$ values usually $>43 \mathrm{~kJ} \mathrm{~mol}^{-1}$ if adsorption of species on the reaction surface and subsequent chemical reaction takes place. Table 5 shows that in case of blank solution, values of $E_{a}$ are smaller than those obtained on adding 12-DHP indicating that 12-DHP affecting, in somehow, the mechanism of solvation. For instance, the acidity (proton availability) is decreased with the addition of an alcohol to a pure acidified aqueous solution. This decrease may be attributed to [8] the gradual breakdown of a quasi-crystalline tetrahedral structure of water caused by interposition of alcohol molecules. Thus, the acidity of the medium decreased due to

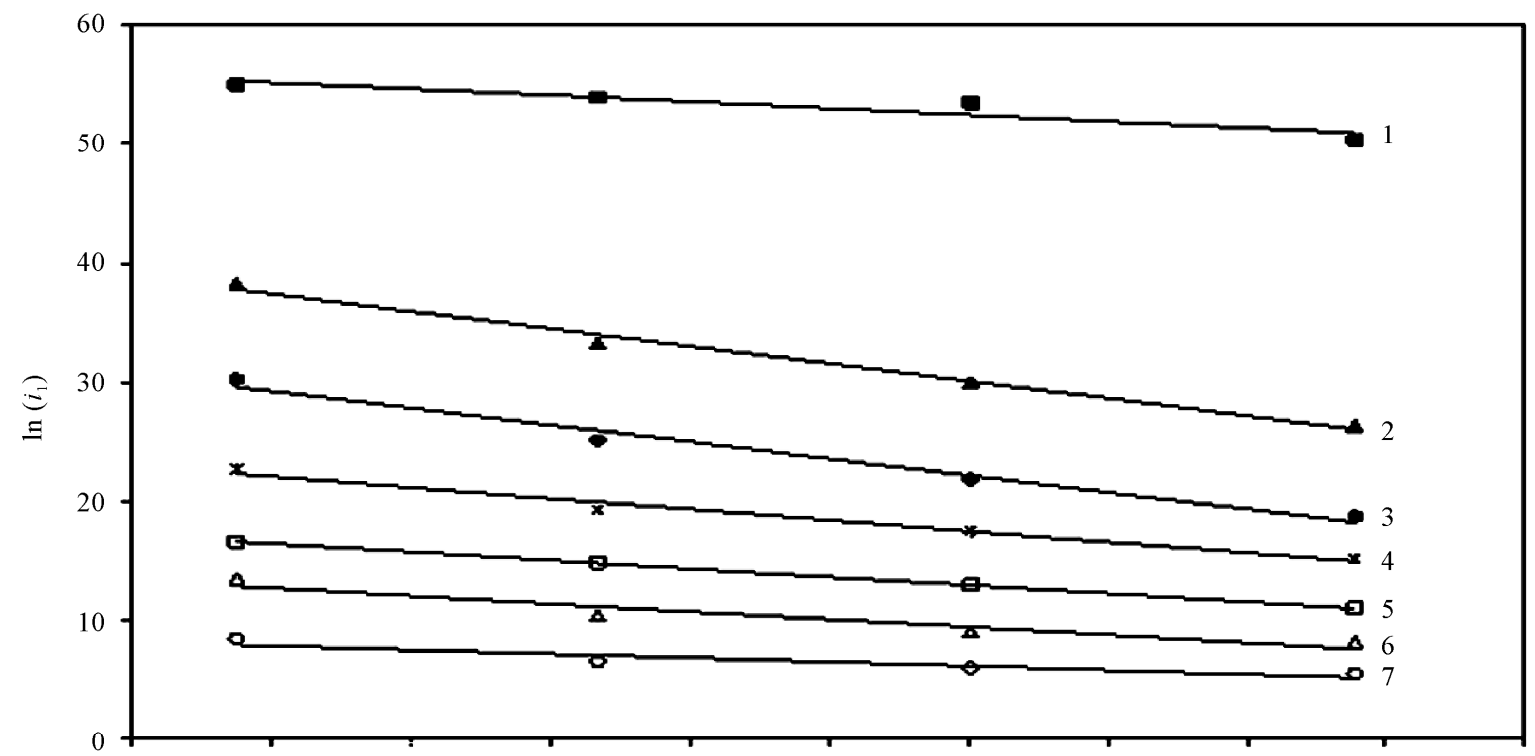

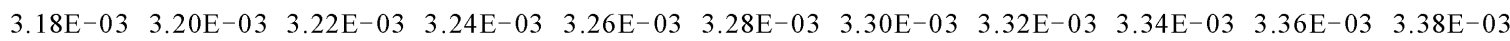

$$
1 / T, \mathrm{~K}^{-1}
$$

Figure 4. Arrhenius plot at 500 rpm. 
Table 5. Electrodeposition average thermodynamic parameters within the temperature range $298 \mathrm{~K}-313 \mathrm{~K}$ for 12-DHP solutions at different speeds of rotation.

\begin{tabular}{|c|c|c|c|c|c|}
\hline & $\mathrm{rpm}$ & $\mathrm{E}_{\mathrm{a}}, \mathrm{kJ} \cdot \mathrm{mol}^{-1}$ & $\mathrm{H}^{*}, \mathrm{~kJ} \cdot \mathrm{mol}^{-1}$ & $\mathrm{~S}^{*}, \mathrm{~J} \cdot \mathrm{mol}^{-1} \cdot \mathrm{K}^{-1}$ & $\mathrm{G}^{*}, \mathrm{~kJ} \cdot \mathrm{mol}^{-1}$ \\
\hline Blank, $0 \%(\mathrm{v} / \mathrm{v}) 12-\mathrm{DHP}$ & & 6.0 & 3.5 & -215.4 & 69.3 \\
\hline $10 \%(\mathrm{v} / \mathrm{v}) 12-\mathrm{DHP}$ & & 11.5 & 9.1 & -199.5 & 69.9 \\
\hline $20 \%(\mathrm{v} / \mathrm{v}) 12-\mathrm{DHP}$ & & 11.4 & 10.3 & -202.2 & 70.7 \\
\hline $30 \%(v / v) 12-D H P$ & 0 & 9.9 & 6.5 & -209.7 & 71.4 \\
\hline $40 \%(\mathrm{v} / \mathrm{v}) 12-\mathrm{DHP}$ & & 11.1 & 7.8 & -208.4 & 72.3 \\
\hline $50 \%(\mathrm{v} / \mathrm{v}) 12-\mathrm{DHP}$ & & 11.2 & 10.4 & -212.1 & 73.4 \\
\hline $60 \%(\mathrm{v} / \mathrm{v}) 12-\mathrm{DHP}$ & & 9.4 & 4.4 & -220.9 & 74.3 \\
\hline Blank, 0\% (v/v) 12-DHP & & 6.3 & 3.8 & -207.1 & 67.1 \\
\hline $10 \%(\mathrm{v} / \mathrm{v}) 12-\mathrm{DHP}$ & & 21.0 & 19.7 & -163.8 & 68.5 \\
\hline $20 \%(\mathrm{v} / \mathrm{v}) 12-\mathrm{DHP}$ & & 27.9 & 13.7 & -143.4 & 69.2 \\
\hline $30 \%(\mathrm{v} / \mathrm{v}) 12-\mathrm{DHP}$ & 100 & 15.8 & 11.2 & -184.5 & 69.6 \\
\hline $40 \%(\mathrm{v} / \mathrm{v}) 12-\mathrm{DHP}$ & & 17.3 & 24.3 & -182.1 & 70.4 \\
\hline $50 \%(\mathrm{v} / \mathrm{v}) 12-\mathrm{DHP}$ & & 15.1 & 20.6 & -191.5 & 71.1 \\
\hline $60 \%(\mathrm{v} / \mathrm{v}) 12-\mathrm{DHP}$ & & 5.3 & 7.0 & -228.3 & 72.5 \\
\hline Blank, 0\% (v/v) 12-DHP & & 8.7 & 6.1 & -196.1 & 66.0 \\
\hline $10 \%(\mathrm{v} / \mathrm{v}) 12-\mathrm{DHP}$ & & 19.1 & 14.8 & -166.8 & 67.5 \\
\hline $20 \%(\mathrm{v} / \mathrm{v}) 12-\mathrm{DHP}$ & & 22.2 & 7.3 & -158.9 & 68.2 \\
\hline $30 \%(v / v) 12-D H P$ & 200 & 20.2 & 20.9 & -167.5 & 68.8 \\
\hline $40 \%(\mathrm{v} / \mathrm{v}) 12-\mathrm{DHP}$ & & 16.9 & 18.4 & -180.0 & 69.3 \\
\hline $50 \%(\mathrm{v} / \mathrm{v}) 12-\mathrm{DHP}$ & & 22.5 & 21.9 & -163.9 & 70.1 \\
\hline $60 \%(\mathrm{v} / \mathrm{v}) 12-\mathrm{DHP}$ & & 17.4 & 2.6 & -185.5 & 71.6 \\
\hline Blank, 0\% (v/v) 12-DHP & & 6.6 & 4.1 & -201.1 & 65.5 \\
\hline $10 \%(\mathrm{v} / \mathrm{v}) 12-\mathrm{DHP}$ & & 18.7 & 18.2 & -166.1 & 66.9 \\
\hline $20 \%(\mathrm{v} / \mathrm{v}) 12-\mathrm{DHP}$ & & 22.5 & 14.3 & -155.9 & 67.6 \\
\hline $30 \%(\mathrm{v} / \mathrm{v}) 12-\mathrm{DHP}$ & 300 & 20.7 & 19.4 & -163.8 & 68.2 \\
\hline $40 \%(\mathrm{v} / \mathrm{v}) 12-\mathrm{DHP}$ & & 18.0 & 17.3 & -175.0 & 69.0 \\
\hline $50 \%(\mathrm{v} / \mathrm{v}) 12-\mathrm{DHP}$ & & 18.2 & 20.7 & -176.4 & 69.5 \\
\hline $60 \%(\mathrm{v} / \mathrm{v}) 12-\mathrm{DHP}$ & & 15.2 & 15.6 & -190.8 & 70.9 \\
\hline Blank, 0\% (v/v) 12-DHP & & 8.2 & 5.6 & -194.2 & 65.0 \\
\hline $10 \%(\mathrm{v} / \mathrm{v}) 12-\mathrm{DHP}$ & & 18.4 & 18.6 & -165.4 & 66.4 \\
\hline $20 \%(\mathrm{v} / \mathrm{v}) 12-\mathrm{DHP}$ & & 24.9 & 13.2 & -146.8 & 67.2 \\
\hline $30 \%(\mathrm{v} / \mathrm{v}) 12-\mathrm{DHP}$ & 400 & 20.8 & 17.9 & -162.1 & 67.8 \\
\hline $40 \%(\mathrm{v} / \mathrm{v}) 12-\mathrm{DHP}$ & & 17.1 & 11.7 & -176.7 & 68.5 \\
\hline $50 \%(\mathrm{v} / \mathrm{v}) 12-\mathrm{DHP}$ & & 17.2 & 22.9 & -178.7 & 69.2 \\
\hline $60 \%(\mathrm{v} / \mathrm{v}) 12-\mathrm{DHP}$ & & 21.8 & 11.9 & -167.2 & 70.4 \\
\hline Blank, $0 \%(\mathrm{v} / \mathrm{v})$ 12-DHP & & 10.2 & 7.7 & -186.6 & 64.7 \\
\hline $10 \%(\mathrm{v} / \mathrm{v}) 12-\mathrm{DHP}$ & 500 & 19.0 & 19.3 & -162.5 & 66.1 \\
\hline $20 \%(\mathrm{v} / \mathrm{v}) 12-\mathrm{DHP}$ & & 24.4 & 14.1 & -147.2 & 66.8 \\
\hline
\end{tabular}




\begin{tabular}{|c|c|c|c|c|c|}
\hline $30 \%(\mathrm{v} / \mathrm{v}) 12-\mathrm{DHP}$ & & 20.3 & 20.7 & -162.6 & 67.5 \\
\hline $40 \%(\mathrm{v} / \mathrm{v}) 12-\mathrm{DHP}$ & & 21.1 & 16.9 & -162.4 & 68.2 \\
\hline $50 \%(\mathrm{v} / \mathrm{v}) 12-\mathrm{DHP}$ & & 25.7 & 17.5 & -150.0 & 69.0 \\
\hline $60 \%(\mathrm{v} / \mathrm{v}) 12-\mathrm{DHP}$ & & 20.9 & 16.5 & -169.3 & 70.1 \\
\hline Blank, 0\% (v/v) 12-DHP & & 8.4 & 5.9 & -191.9 & 64.5 \\
\hline $10 \%(\mathrm{v} / \mathrm{v}) 12-\mathrm{DHP}$ & & 18.6 & 18.5 & -162.7 & 65.8 \\
\hline $20 \%(\mathrm{v} / \mathrm{v}) 12-\mathrm{DHP}$ & & 21.7 & 13.3 & -155.3 & 66.6 \\
\hline $30 \%(\mathrm{v} / \mathrm{v}) 12-\mathrm{DHP}$ & 600 & 21.1 & 21.8 & -159.1 & 67.2 \\
\hline $40 \%(\mathrm{v} / \mathrm{v}) 12-\mathrm{DHP}$ & & 21.0 & 18.9 & -161.9 & 67.9 \\
\hline $50 \%(\mathrm{v} / \mathrm{v}) 12-\mathrm{DHP}$ & & 29.7 & 26.6 & -136.2 & 68.8 \\
\hline $60 \%(\mathrm{v} / \mathrm{v}) 12-\mathrm{DHP}$ & & 24.2 & 28.0 & -157.6 & 69.8 \\
\hline Blank, $0 \%(\mathrm{v} / \mathrm{v})$ 12-DHP & & 3.9 & 1.3 & -206.1 & 64.3 \\
\hline $10 \%(\mathrm{v} / \mathrm{v}) 12-\mathrm{DHP}$ & & 19.5 & 18.4 & -159.1 & 65.5 \\
\hline $20 \%(\mathrm{v} / \mathrm{v}) 12-\mathrm{DHP}$ & & 27.4 & 16.4 & -135.4 & 66.3 \\
\hline $30 \%(\mathrm{v} / \mathrm{v}) 12-\mathrm{DHP}$ & 700 & 20.2 & 18.5 & -161.5 & 67.0 \\
\hline $40 \%(\mathrm{v} / \mathrm{v}) 12-\mathrm{DHP}$ & & 18.7 & 17.6 & -168.9 & 67.8 \\
\hline $50 \%(\mathrm{v} / \mathrm{v}) 12-\mathrm{DHP}$ & & 27.5 & 31.8 & -142.5 & 68.5 \\
\hline $60 \%(\mathrm{v} / \mathrm{v}) 12-\mathrm{DHP}$ & & 29.2 & 30.3 & -140.1 & 69.5 \\
\hline Blank, 0\% (v/v) 12-DHP & & 14.4 & 11.8 & -170.7 & 64.0 \\
\hline $10 \%(\mathrm{v} / \mathrm{v}) 12-\mathrm{DHP}$ & & 22.0 & 20.3 & -150.2 & 65.3 \\
\hline $20 \%(\mathrm{v} / \mathrm{v}) 12-\mathrm{DHP}$ & & 24.9 & 17.3 & -143.2 & 66.1 \\
\hline $30 \%(\mathrm{v} / \mathrm{v}) 12-\mathrm{DHP}$ & 800 & 19.5 & 20.7 & -163.1 & 66.8 \\
\hline $40 \%(\mathrm{v} / \mathrm{v}) 12-\mathrm{DHP}$ & & 20.4 & 23.1 & -162.5 & 67.5 \\
\hline $50 \%(\mathrm{v} / \mathrm{v}) 12-\mathrm{DHP}$ & & 27.5 & 33.3 & -142.1 & 68.3 \\
\hline $60 \%(\mathrm{v} / \mathrm{v}) 12-\mathrm{DHP}$ & & 26.8 & 26.4 & -146.8 & 69.1 \\
\hline Blank, 0\% (v/v) 12-DHP & & 18.3 & 15.7 & -157.0 & 63.7 \\
\hline $10 \%(\mathrm{v} / \mathrm{v}) 12-\mathrm{DHP}$ & & 23.7 & 19.5 & -144.0 & 65.2 \\
\hline $20 \%(\mathrm{v} / \mathrm{v}) 12-\mathrm{DHP}$ & & 31.2 & 23.1 & -121.8 & 65.9 \\
\hline $30 \%(\mathrm{v} / \mathrm{v}) 12-\mathrm{DHP}$ & 900 & 22.0 & 20.6 & -154.3 & 66.6 \\
\hline $40 \%(\mathrm{v} / \mathrm{v}) 12-\mathrm{DHP}$ & & 24.0 & 22.5 & -150.2 & 67.4 \\
\hline $50 \%(\mathrm{v} / \mathrm{v}) 12-\mathrm{DHP}$ & & 27.5 & 33.4 & -141.5 & 68.1 \\
\hline $60 \%(\mathrm{v} / \mathrm{v}) 12-\mathrm{DHP}$ & & 28.0 & 33.3 & -142.5 & 69.0 \\
\hline Blank, 0\% (v/v) 12-DHP & & 17.6 & 15.0 & -158.7 & 63.5 \\
\hline $10 \%(\mathrm{v} / \mathrm{v}) 12-\mathrm{DHP}$ & & 20.1 & 20.2 & -155.3 & 65.0 \\
\hline $20 \%(\mathrm{v} / \mathrm{v}) 12-\mathrm{DHP}$ & & 31.7 & 20.6 & -119.3 & 65.7 \\
\hline $30 \%(\mathrm{v} / \mathrm{v}) 12-\mathrm{DHP}$ & 1000 & 20.2 & 20.1 & -159.8 & 66.5 \\
\hline $40 \%(\mathrm{v} / \mathrm{v}) 12-\mathrm{DHP}$ & & 23.1 & 25.3 & -152.6 & 67.2 \\
\hline $50 \%(\mathrm{v} / \mathrm{v}) 12-\mathrm{DHP}$ & & 24.7 & 35.1 & -149.9 & 68.0 \\
\hline $60 \%(\mathrm{v} / \mathrm{v}) 12-\mathrm{DHP}$ & & 26.6 & 30.3 & -146.3 & 68.8 \\
\hline
\end{tabular}


the change in the state of solvation. Table 5 shows also that all $\mathrm{E}_{\mathrm{a}}$ values are lower than $43 \mathrm{~kJ} \mathrm{~mol}^{-1}$; characterizing diffusion processes are controlling the electrodeposition reaction. Figure 5 is another presentation of controlling the crystallite size as a parameter of two variables, the composition of 12-DHP and the speed of rotation. As it is shown in Figure 5, the highest composition of 12-DHP does not mean the lowest $\mathrm{E}_{\mathrm{a}}$ value and vice versa as well as does the same meaning on the speed of rotation. From Table 5, it is also noticed that the weak dependence of $\Delta \mathrm{G}^{*}$ on the composition of the organic solvent can be attributed largely to the general linear compensation between $\Delta \mathrm{H}^{*}$ and $\Delta \mathrm{S}^{*}$ for the given temperature.

The angular velocity, $\omega$, is given by Equation (8). Figure 6 gives the relation between the limiting current density and the angular velocity, $\omega$, to the power 0.7 at different compositions of 12-DHP and at $298 \mathrm{~K}$ as a representative graph. In Figure 6, straight lines were obtained and the limiting current density increases by increasing rotation, which indicates that the electrodeposition reaction is diffusion-controlled reaction [11].

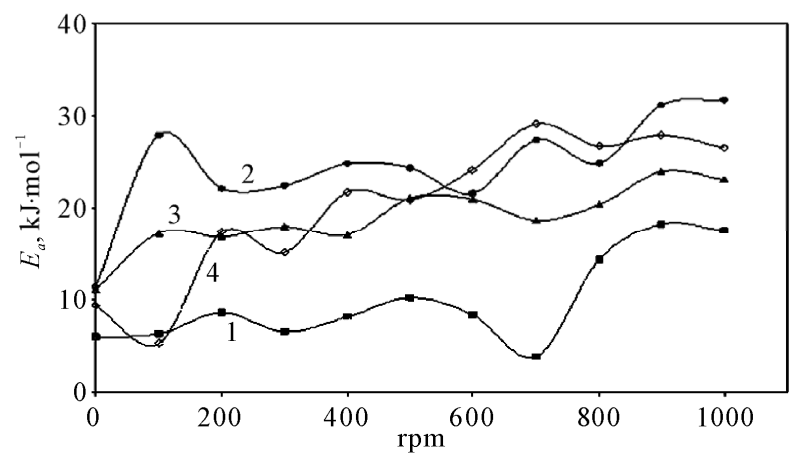

Figure 5. Variation of activation energy, $E_{a}$, with the speed of rotation, rpm, for (1) Blank, (2) $20 \%$ (v/v) 12-DHP, (3) $40 \%$ (v/v) 12-DHP, (4) $60 \%$ (v/v) 12-DHP.

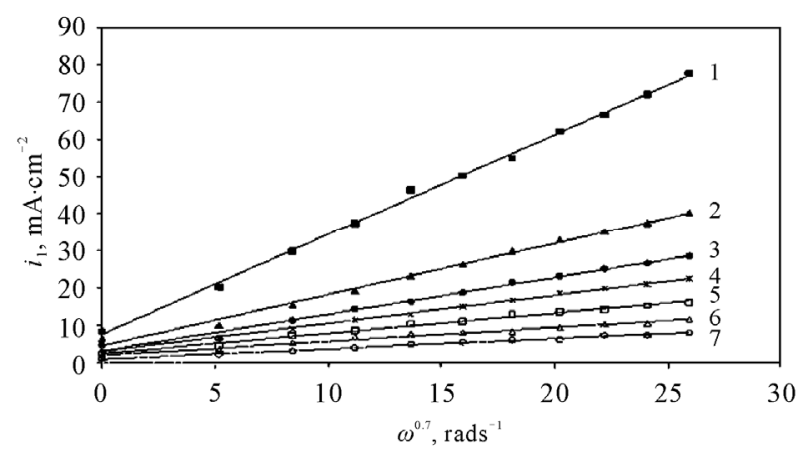

Figure 6. Variation of the limiting current density, $i_{l}$, with the angular velocity to a power $0.7, \omega^{0.7}$, at $298 \mathrm{~K}$ for (1) Blank, (2) $10 \%$ (v/v) 12-DHP, (3) $20 \%$ (v/v) 12-DHP, (4) $30 \%$ (v/v) 12-DHP, (5) $40 \%$ (v/v) 12-DHP, (6) $50 \%$ (v/v) 12-DHP, (7) $60 \%$ (v/v) 12-DHP.

$$
\omega=\frac{2 \pi r p m}{60}
$$

The diffusion coefficient of $\mathrm{Cu}^{2+}$ ions, $\mathrm{D}$, in different solutions was determined from the values of limiting current densities using Eisenberg equation [14]:

$$
i_{l}=0.0791 n F C_{b} U^{0.7} d_{i}^{-0.3} v^{-0.344} D^{0.644}
$$

where $C_{b}$ is the bulk concentration $\left(\mathrm{mol} \cdot \mathrm{cm}^{-3}\right), U$ is the peripheral velocity $=\omega r$ in $\mathrm{cm} \cdot \mathrm{rad} \cdot \mathrm{s}^{-1}(r$ is the radial distance in $\mathrm{cm}$ ) or $U=2 \pi \omega r$ in $\mathrm{cm} \cdot \mathrm{s}^{-1}, \mathrm{~d}$ is the characteristic length for the rotating cylinder in $\mathrm{cm}$, and $v$ is the kinematic viscosity in stoke $(v=\eta / \rho, \eta$ is the viscosity in $\mathrm{g} \cdot \mathrm{cm}^{-1} \cdot \mathrm{s}^{-1}$ and $\rho$ is the density in $\left.\mathrm{g} \cdot \mathrm{cm}^{-3}\right)$.

The diffusion coefficient; $D$, of $\mathrm{Cu}^{2+}$ ions in solutions containing alcohols decreases due to the increase in the interfacial viscosity [15], $\eta$, in accordance with the Stokes-Einstein equation:

$$
\frac{\eta D}{T}=\text { const } \text {. }
$$

To obtain an overall mass transfer correlation under the present conditions by using the method of dimensional analysis we suppose:

$$
S h=a R e^{b} \cdot S c^{c}
$$

where: $S h, R e$ and $S c$ are the Sherwood $(S h=k 1 / D)$, Reynolds $(\operatorname{Re}=1 U / v)$ and Schmidt $(S c=v / D)$ numbers; respectively and $\mathrm{a}$ and $\mathrm{b}$ are empirical constants, $c=0.33$ (indicating forced convection).

Table 6 summarizes the values of dimensionless groups ( $S h, S c$ and $R e$ ) at $298 \mathrm{~K}$ as an example of the data. By plotting $\log \left(S h / S c^{0.33}\right)$ against $\log (R e)$, a straight line was obtained its slope gave the constant $b$ while the intercept gives the other constant a. Figure 7 shows the overall correlation at all experimental variables. Constants $a$ and $b$ were extracted by applying Figure 7 on the data and the values are given in Table 7. The present results agree with the polarographic studies $[16,17]$ conducted in solutions containing surfactants where it was found that the diffusion current decreases in

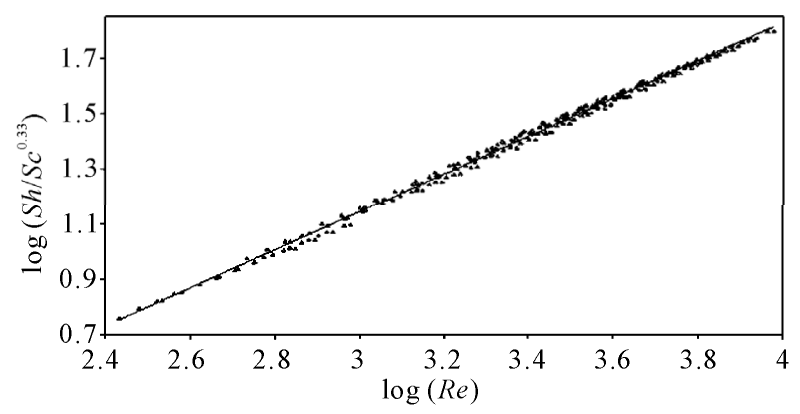

Figure 7. Overall mass transfer correlation under the present experimental variables. 
Table 6. Dimensionless groups used in dimensional analysis for all solutions at $298 \mathrm{~K}$.

\begin{tabular}{|c|c|c|c|c|}
\hline & rpm & $S h=k l / D$ & $S c=v / D$ & $R e=1 U / v$ \\
\hline \multirow{14}{*}{ Blank, 0\% (v/v) 12-DHP } & 100 & 90 & 737 & 705 \\
\hline & 200 & 155 & 858 & 1409 \\
\hline & 300 & 212 & 941 & 2114 \\
\hline & 400 & 257 & 914 & 2818 \\
\hline & 500 & 314 & 1029 & 3523 \\
\hline & 600 & 364 & 1093 & 4228 \\
\hline & 700 & 402 & 1068 & 4932 \\
\hline & 800 & 448 & 1110 & 5637 \\
\hline & 900 & 487 & 1117 & 6342 \\
\hline & 1000 & 524 & 1115 & 7046 \\
\hline & 100 & 130 & 2206 & 681 \\
\hline & 200 & 218 & 2415 & 1362 \\
\hline & 300 & 301 & 2671 & 2042 \\
\hline & 400 & 371 & 2743 & 2723 \\
\hline \multirow{10}{*}{$10 \%(\mathrm{v} / \mathrm{v}) 12-\mathrm{DHP}$} & 500 & 441 & 2859 & 3404 \\
\hline & 600 & 501 & 2859 & 4085 \\
\hline & 700 & 562 & 2925 & 4765 \\
\hline & 800 & 626 & 3048 & 5446 \\
\hline & 900 & 686 & 3125 & 6127 \\
\hline & 1000 & 741 & 3148 & 6808 \\
\hline & 100 & 155 & 4669 & 594 \\
\hline & 200 & 240 & 4131 & 1189 \\
\hline & 300 & 329 & 4494 & 1783 \\
\hline & 400 & 419 & 5014 & 2377 \\
\hline \multirow{10}{*}{$20 \%(\mathrm{v} / \mathrm{v}) 12-\mathrm{DHP}$} & 500 & 494 & 5163 & 2971 \\
\hline & 600 & 558 & 5081 & 3566 \\
\hline & 700 & 633 & 5337 & 4160 \\
\hline & 800 & 701 & 5460 & 4754 \\
\hline & 900 & 771 & 5659 & 5348 \\
\hline & 1000 & 832 & 5701 & 5943 \\
\hline & 100 & 140 & 4784 & 508 \\
\hline & 200 & 252 & 6410 & 1016 \\
\hline & 300 & 342 & 6820 & 1524 \\
\hline & 400 & 436 & 7636 & 2032 \\
\hline \multirow[t]{5}{*}{$30 \%(\mathrm{v} / \mathrm{v}) 12-\mathrm{DHP}$} & 500 & 511 & 7692 & 2540 \\
\hline & 600 & 590 & 8072 & 3048 \\
\hline & 700 & 657 & 8060 & 3556 \\
\hline & 800 & 734 & 8466 & 4064 \\
\hline & 900 & 810 & 8858 & 4572 \\
\hline
\end{tabular}




\begin{tabular}{|c|c|c|c|c|}
\hline \multirow{11}{*}{$40 \%(\mathrm{v} / \mathrm{v}) 12-\mathrm{DHP}$} & 1000 & 873 & 8875 & 5080 \\
\hline & 100 & 154 & 9167 & 420 \\
\hline & 200 & 254 & 9512 & 840 \\
\hline & 300 & 361 & 11499 & 1261 \\
\hline & 400 & 446 & 11821 & 1681 \\
\hline & 500 & 551 & 13821 & 2101 \\
\hline & 600 & 615 & 13180 & 2521 \\
\hline & 700 & 705 & 14244 & 2941 \\
\hline & 800 & 795 & 15357 & 3362 \\
\hline & 900 & 870 & 15694 & 3782 \\
\hline & 1000 & 948 & 16254 & 4202 \\
\hline \multirow{9}{*}{$50 \%(\mathrm{v} / \mathrm{v}) 12-\mathrm{DHP}$} & 100 & 156 & 14145 & 342 \\
\hline & 200 & 271 & 17071 & 684 \\
\hline & 300 & 365 & 17816 & 1026 \\
\hline & 400 & 472 & 20842 & 1368 \\
\hline & 500 & 583 & 24300 & 1710 \\
\hline & 600 & 701 & 28481 & 2052 \\
\hline & 700 & 774 & 27863 & 2394 \\
\hline & 800 & 851 & 27930 & 2736 \\
\hline & 900 & 953 & 30459 & 3078 \\
\hline \multirow{11}{*}{$60 \%(\mathrm{v} / \mathrm{v}) 12-\mathrm{DHP}$} & 1000 & 1020 & 29928 & 3419 \\
\hline & 100 & 171 & 29090 & 271 \\
\hline & 200 & 315 & 41288 & 543 \\
\hline & 300 & 437 & 46559 & 814 \\
\hline & 400 & 527 & 44784 & 1086 \\
\hline & 500 & 637 & 49127 & 1357 \\
\hline & 600 & 731 & 50485 & 1629 \\
\hline & 700 & 859 & 58635 & 1900 \\
\hline & 800 & 904 & 52160 & 2172 \\
\hline & 900 & 1028 & 59284 & 2443 \\
\hline & 1000 & 1098 & 57975 & 2715 \\
\hline
\end{tabular}

Table 7. Dimensionless correlation constants $a$ and $b$ under experimental conditions.

\begin{tabular}{|c|c|c|c|c|c|c|c|c|}
\hline & \multicolumn{4}{|c|}{ Constant $a$} & \multicolumn{4}{|c|}{ Constant $b$} \\
\hline & 298 K & $303 \mathrm{~K}$ & $308 \mathrm{~K}$ & 313 K & 298 K & $303 \mathrm{~K}$ & $308 \mathrm{~K}$ & $313 \mathrm{~K}$ \\
\hline Blank & 0.1005 & 0.0990 & 0.0998 & 0.1017 & 0.7048 & 0.7062 & 0.7049 & 0.7018 \\
\hline $10 \%$ 12-DHP & 0.1039 & 0.1030 & 0.1029 & 0.1021 & 0.7040 & 0.7043 & 0.7037 & 0.7037 \\
\hline 20\% 12-DHP & 0.1064 & 0.1030 & 0.1032 & 0.1047 & 0.7031 & 0.7062 & 0.7049 & 0.7019 \\
\hline $30 \% 12-\mathrm{DHP}$ & 0.1047 & 0.1040 & 0.1038 & 0.1034 & 0.7065 & 0.7064 & 0.7059 & 0.7054 \\
\hline $40 \%$ 12-DHP & 0.1060 & 0.1040 & 0.1050 & 0.1051 & 0.7070 & 0.7084 & 0.7062 & 0.7053 \\
\hline $50 \%$ 12-DHP & 0.1058 & 0.1063 & 0.1057 & 0.1069 & 0.7094 & 0.7077 & 0.7074 & 0.7049 \\
\hline $60 \%$ 12-DHP & 0.1098 & 0.1134 & 0.1147 & 0.1139 & 0.7072 & 0.7018 & 0.6991 & 0.6994 \\
\hline
\end{tabular}


the presence of surfactants.

Overall, the data in this investigation were valid for 90 $<S h<1098,737<S c<59284$ and $271<R e<7046$. In addition, the results fit the theme of a forced convection mechanism, which agrees very well with our previous study on the electrorefining of copper [2].

\subsection{Morphology and Composition Analyses}

The morphology of electrodeposited copper from acidic baths on poly or single crystal copper electrodes with and/or without additives has been widely studied $[2,18$, 19] and the results in this work were essentially similar. Since the concern was mainly the extraction and production of pure copper, all experiments were proceeded at the limiting current density that was determined for each solution and with time range $10-30$ minutes, so it is expected that the morphologies of electrodeposited copper must be characterized by 3-D nucleation [20]. Concerning the type of solution with and/or without 12-DHP at different deposition time-periods, no unusual behaviour has occurred and the rate of copper electrodeposition increases by increasing time and reflects the same trend of the limiting current density. As for the influence of different amounts of 12-DHP, copper was deposited in very smooth and compact form up to $30 \%(\mathrm{v} / \mathrm{v}) 12$-DHP as shown in Figure 8(a). The deposited copper from solutions of $40 \%-60 \%(\mathrm{v} / \mathrm{v}) 12$-DHP was rough and discontinuous as shown in Figure 8(b), which gives an idea about the behaviour of 12-DHP with its composition. Also, this compositional effect of 12-DHP confines with and proves the hypothesis of the departure percent, S. Regarding the temperature effect, and as stated that temperature is an important factor in the electrodeposition of metals [20]. The main features of the surface do not change with changing the temperature, only the deposit roughness as well as the crystallite size increases with increasing temperature.

High current densities or high cathodic overpotentials, accompanied by fast stirring rates can adversely affect electrodeposition process resulting in brittle deposits, which can comprise occlusions of additives and foreign materials [21]. Although, it was stated [22] that an increase of copper ion concentration or agitation increases the size of the particles and obscured their dendritic character, it was found dramatic changes occurred when rotation started, dendrite nodules were formed with high degree of discontinuity as well as very rough deposits as in case of 10\% (v/v) 12-DHP solution at $200 \mathrm{rpm}$, Figure 9(a). The phenomenon of dendritic growth [23] may occur if the rate of the electrodeposition process is partially diffusion controlled, so, on a microrough surface,

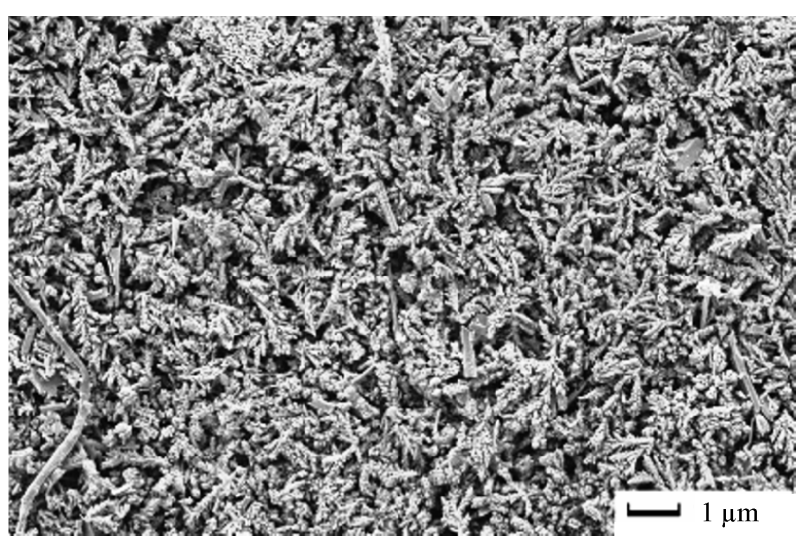

(a)

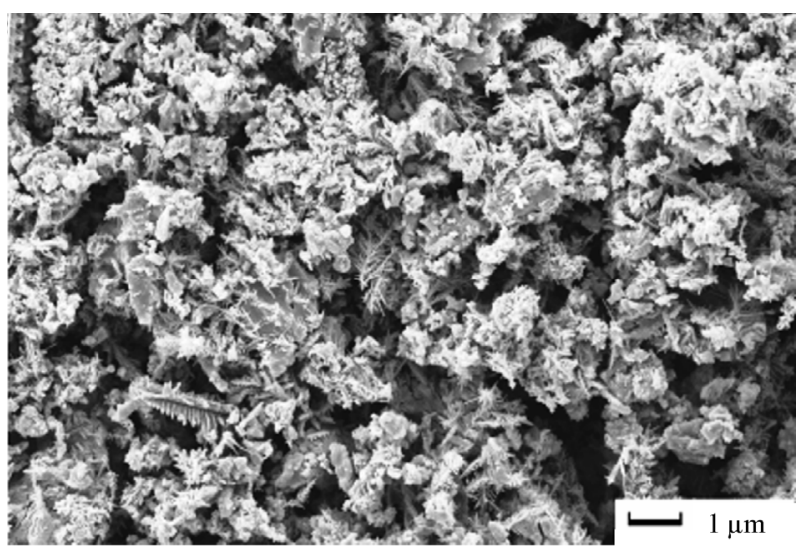

(b)

Figure 8. SEM micrographs of electrodeposited copper powder after 20 minutes at $298 \mathrm{~K}$ and zero rpm from (a) $30 \%$ (v/v) 12-DHP, (b) 40 - $60 \%$ (v/v) 12-DHP.

the rate of deposition at surface peaks will be greater than that at troughs. These surface non-uniformities therefore become more pronounced resulting, eventually, in the formation of macrorough deposits. The phenomenon of dendritic growth may also be related to differences in the rate of diffusion at different points on a surface. In the case of macrospiral growth, for instance, spherical diffusion to the tip of the spiral results in a higher rate of mass transport to this point and this may lead to dendritic growth. Contrarily to Figure 9(a) at 200 $\mathrm{rpm}$, grape-like aggregates were formed with low degree of discontinuity at $1000 \mathrm{rpm}$ in case of $10 \%(\mathrm{v} / \mathrm{v}) 12$-DHP solution, Figure 9(b).

Figure 10 gives the EDS analysis for some chosen specimens that represent most of the experimental conditions, which have been studied. The analysis indicated that the composition of the electrodeposited copper powder under different conditions for all blank solutions was mainly pure copper (94.56 at. \%) and oxygen (5.44 at. \%), while it was around 99 at. \% copper and less than 1 at. $\%$ oxygen for all 12-DHP solutions. Tiny percent 


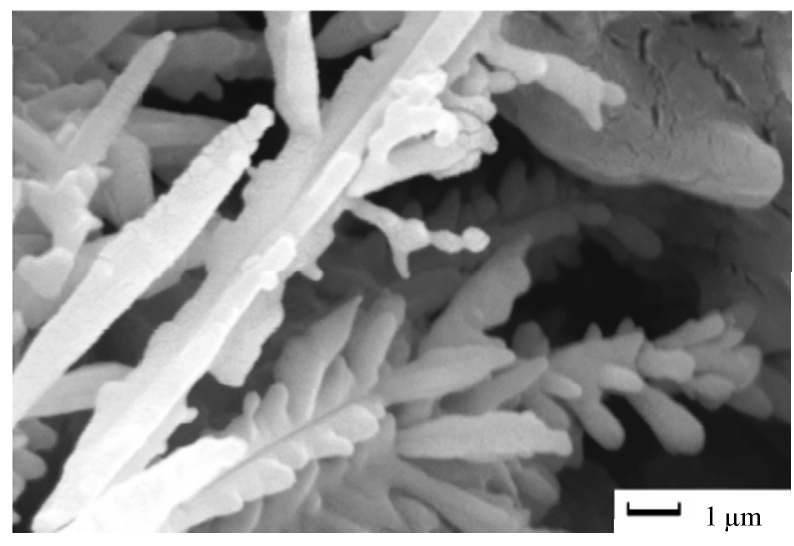

(a)

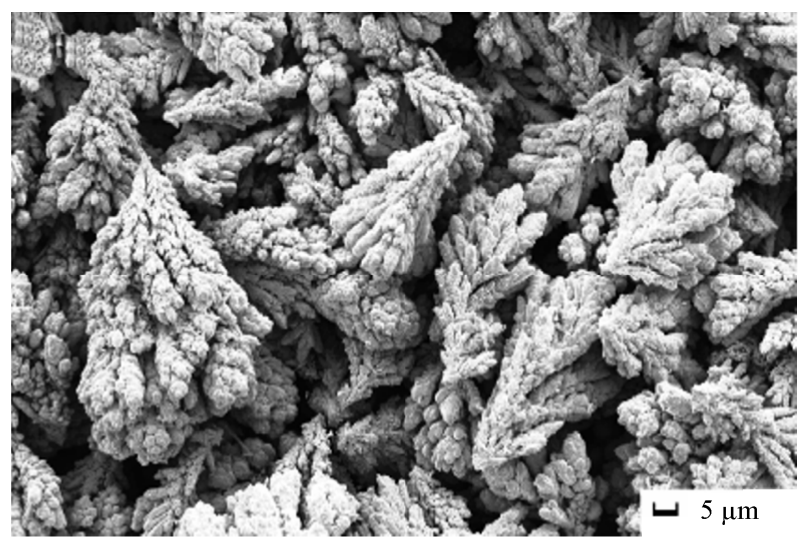

(b)

Figure 9. SEM micrographs of electrodeposited copper powder after 20 minutes from $10 \%$ (v/v) 12-DHP at $298 \mathrm{~K}$ and at (a) $200 \mathrm{rpm}$, (b) $1000 \mathrm{rpm}$.

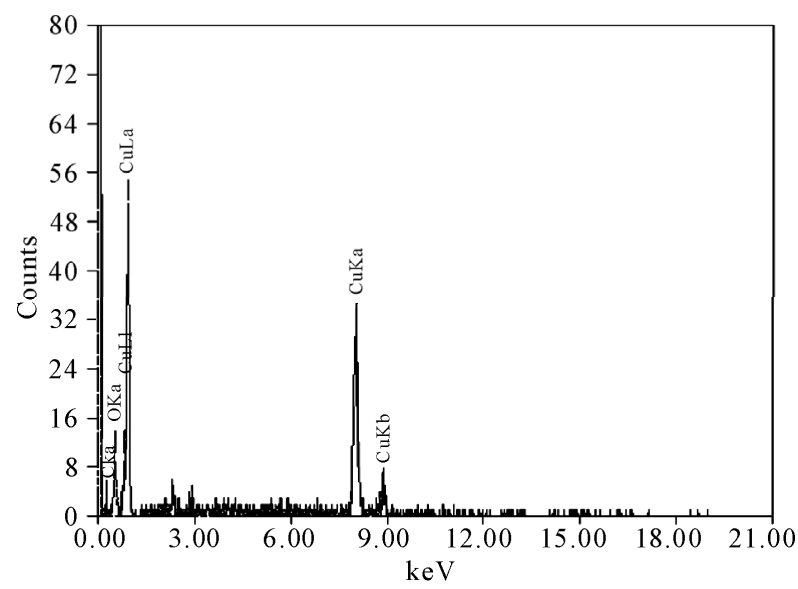

Figure 10. Deposit average EDS analysis.

age of silicon and carbon were present, which may be due to the occlusion of foreign materials [21], on one hand. On the other hand, the presence of silicon could be attributable to the attendance of some glass leftover from another specimen, while carbon is referable to the coat- ing of specimen with carbon before the analysis. In addition, oxygen peak in Figure 10 might caused by the oxidation of a negligible part of the deposit due to the high voltage used in the analysis. Figure 11 represents the distribution of both copper and oxygen in the produced powder under different conditions, showing the mutual homogeneous distribution of both copper and oxygen.

XRD patterns of the deposited copper powder are presented in Figure 12, which summarizes all the three possible orientations in this study. This exhibits polycrystalline copper as deposited state with (111) as prominent plane parallel to the substrate. According to the standard PDF card (No. 04-0836) [2], all of the detected peaks are indexed as those from the cubic crystal [space group Fm3m (225)] with small two peaks that patterned for $\mathrm{Cu}_{2} \mathrm{O}$. Almost single orientation (111) was detected in case of $10-30 \%(\mathrm{v} / \mathrm{v})$ 12-DHP, while three different orientations $(111,200$ and 220) are obviously present when $40 \%$ - 60\% (v/v) 12-DHP was added to the deposition mixture. The average crystallite size was calculated using Scherrer's equation:

$$
C S=\frac{0.9 \lambda}{B \cos \theta_{B}}
$$

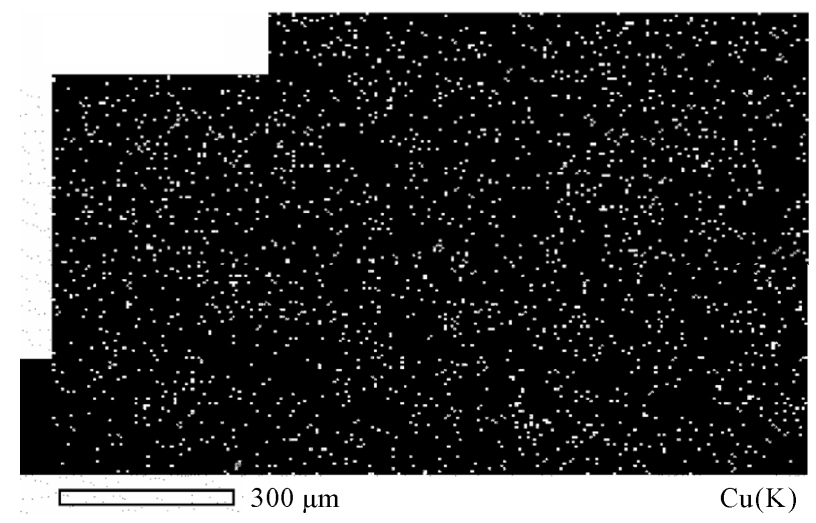

(a)

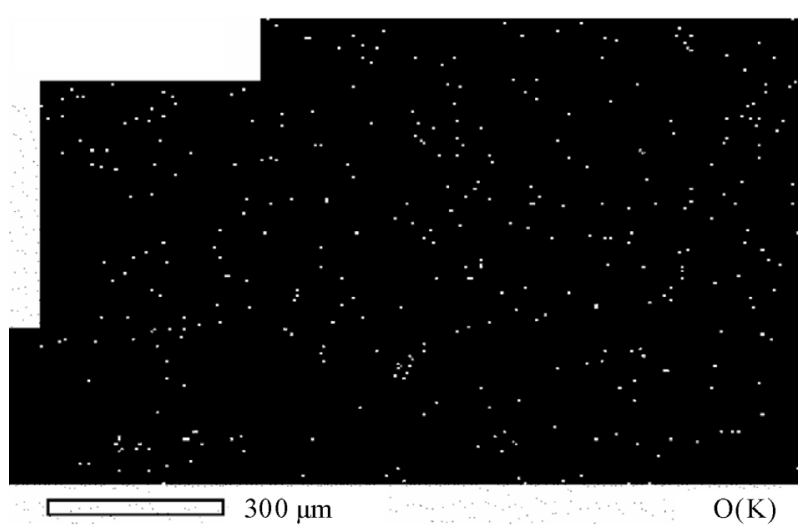

(b)

Figure 11. Atomic distribution of a) $\mathrm{Cu}(\mathrm{K})$ and $b) \mathrm{O}(\mathrm{K})$. 


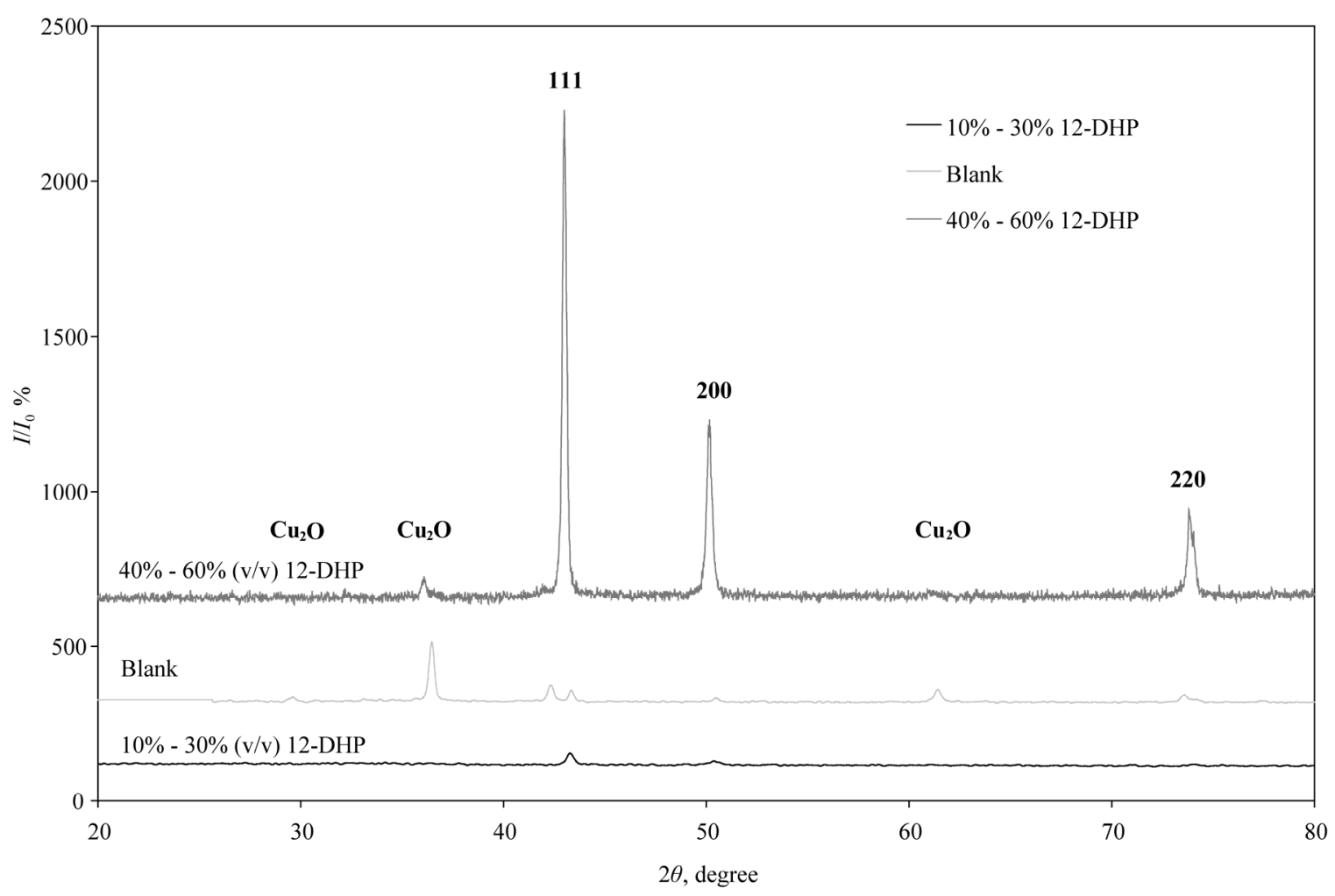

Figure 12. XRD patterns of all possible orientations for as deposited $\mathrm{Cu}$ powder at different experimental conditions.

where $C S$ is the crystallite size in $\mathrm{nm}, \lambda$ is the wave length of X-ray radiation source $\left(\mathrm{Cu} \mathrm{K} \alpha_{1}, \lambda=1.54056\right)$, $\mathrm{B}$ is the full-width at half maximum and $\theta$ is the angle.

The average crystallite size of copper powder electrodeposited in absence of 12-DHP was ranged between $177.80 \mathrm{~nm}$ (at $298 \mathrm{~K}$ and $1000 \mathrm{rpm}$ ) and $10.04 \mathrm{~nm}$ (at $298 \mathrm{~K}$ and $0 \mathrm{rpm})$. In presence of $10 \%-30 \%(\mathrm{v} / \mathrm{v})$ 12-DHP the average crystallite size range was $250.6 \mathrm{~nm}$ (at $298 \mathrm{~K}$ and $1000 \mathrm{rpm}$ ) $-12.43 \mathrm{~nm}$ (at $298 \mathrm{~K}$ and 0 rpm), whereas it was $140.95 \mathrm{~nm}$ (at $298 \mathrm{~K}$ and $1000 \mathrm{rpm}$ ) $-7.14 \mathrm{~nm}$ (at $298 \mathrm{~K}$ and $0 \mathrm{rpm}$ ) on using $40 \%-60 \%$ (v/v) 12-DHP. Figure 13 shows the change of crystallite size; $\mathrm{CS}$; with variation in the angular velocity; $\omega$. A linear relationship was deduced in case of blank solution (equation 13), while exponential equations 14 and 15 govern the same relationship in case of $10 \%-30 \%(\mathrm{v} / \mathrm{v})$ 12-DHP and 40\% - 60\% (v/v) 12-DHP; respectively.

$$
\begin{gathered}
C S=10.617+1.5507 \varpi \\
C S=10.957 \mathrm{e}^{0.029 \varpi} \\
C S=8.3583 \mathrm{e}^{0.0283 \pi}
\end{gathered}
$$

\section{Conclusions}

From the present study concerning the electrowinning process of copper from acidified copper sulphate solu- tions and in the presence of 12-DHP using the rotating cylinder electrode (RCE) the data were valid for $90<\mathrm{Sh}$ $<1098,737<\mathrm{Sc}<59284$ and $271<\operatorname{Re}<7046$ and the results agreed with the previous studies of mass transfer to rotating cylinders in turbulent flow systems. Addition of 12-DHP to the sulphate electrodeposition bath increases the cathodic polarisation and decreases the value of the limiting current density, while it increases with increasing temperature and the speed of rotation. An empirical parameter called the departure percent, S, was obtained which may represent the stability of the organic additive in the given medium and under the experimental conditions. The percentage of inhibition, $\mathrm{P}$, was in the range $0.00-89.91 \%$ depending on the concentration of 12-DHP as well as the temperature. Values of activation energy of electrodeposition process, $E_{a}$, were found to be less than $28 \mathrm{~kJ} \mathrm{~mol}^{-1}$ indicating diffusion controlled process. Various crystallite sizes ranged $7.1 \mathrm{~nm}-250.6$ $\mathrm{nm}$ were obtained with three size categories of blank, $10 \%$ - $30 \%(\mathrm{v} / \mathrm{v}) 12-\mathrm{DHP}$ and $40 \%$ - 60\% (v/v) 12-DHP solutions. The EDS elemental analysis and XRD diffraction patterns proved that the formed powders were pure copper with tiny percent of oxygen, silicon and carbon.

\section{Acknowledgements}

The authors gratefully acknowledge the staff of Mubarak 


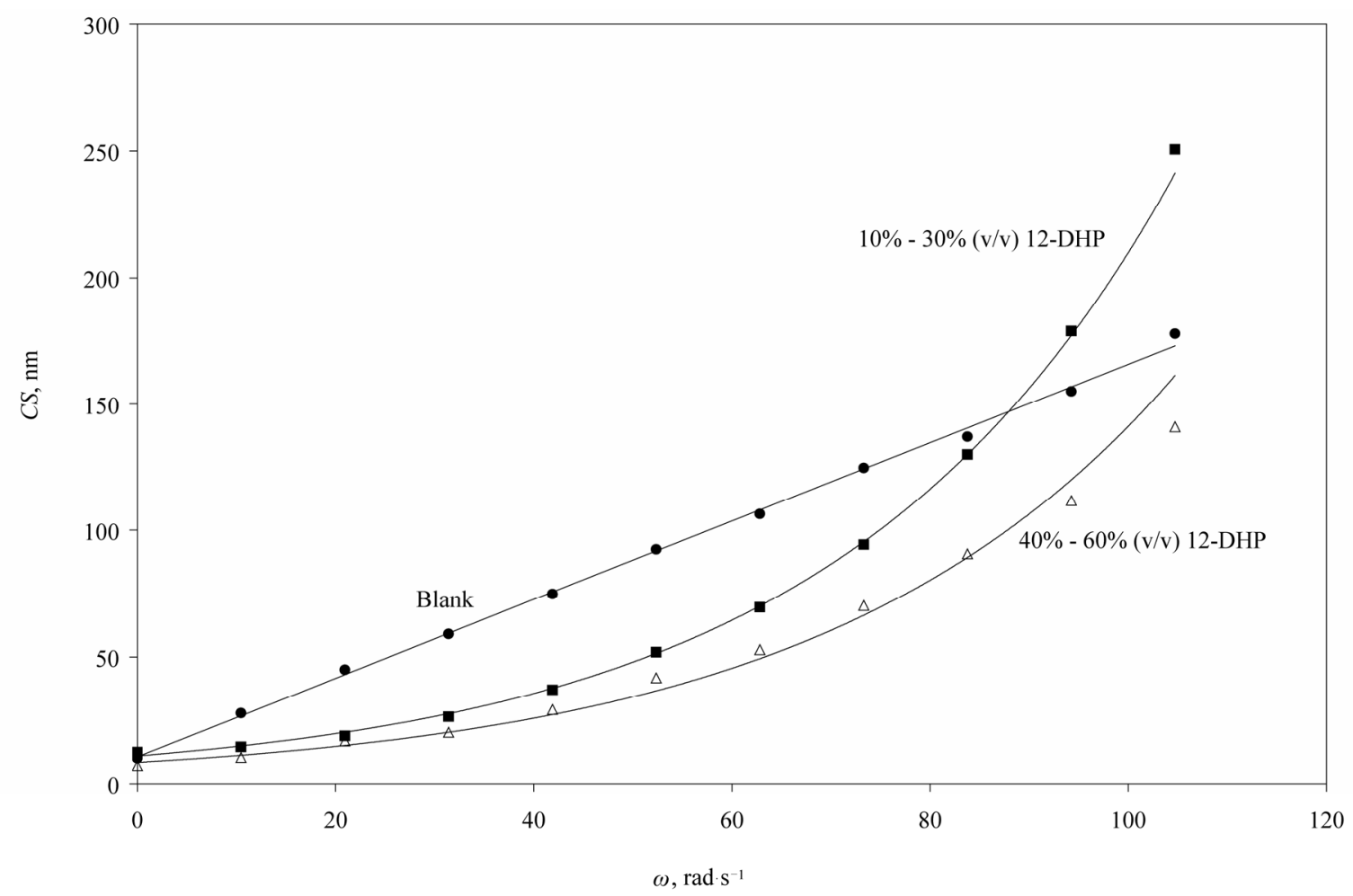

Figure 13. Variation of the crystallite size, CS, with the angular velocity, $\omega$, at $298 \mathrm{~K}$ showing the three possible correlations in this study.

city for scientific research and technology applications (MuCSAT) for funding this work among the internal project of electrochemistry and nanotechnology. Also, the acknowledgement goes to the Egyptian Science and Technology Development Fund (STDF) for funding this work among the STDF project No. 277.

\section{References}

[1] J. L. Sigley, P. C. Johnson and S. P. Beaudoin, "Use of Nonionic Surfactant to Reduce Sulfuric Acid Mist in the Copper Electrowinning Process," Hydrometallurgy, Vol. 70, No. 1-3, July 2003, pp. 1-8. doi:10.1016/S0304-386X(03)00077-X|

[2] H. M. A. Soliman and H. H. Abdel-Rahman, "The Use of Rotating Cylinder Electrode to Study the Effect of 1,3-Dihydroxypropane on the Production of Copper Powder," Journal of the Brazilian Chemical Society, Vol. 17, No. 4, 2006, pp. 705-714. doi:10.1590/S0103-50532006000400011

[3] M. Alodan and W. Smyrl, "Effect of Thiourea on Copper Dissolution and Deposition," Electrochim Acta, Vol. 44, No. 2-3, September 1998, pp. 299-309. doi:10.1016/S0013-4686(98)00060-7

[4] Z. D. Stankovic, "Effect of Gelatine and Thiourea on the Kinetics and Mechanism of Anodic Dissolution and Cathodic Deposition of Copper," Erzmetall, Vol. 38, No. 7-8, July 1985, pp. 361-365.
[5] L. Muresan, S. Varvara, G. Maurin and S. Dorneanu, "The Effect of Some Organic Additives upon Copper Electrowinning from Sulphate Electrolytes," Hydrometallurgy, Vol. 54, No. 2-3, January 2000, pp. 161-169. doi:10.1016/S0304-386X(99)00063-8

[6] J. Vereecken and R. Winand, "Influence of Polyacrylamides on the Quality of Copper Deposits from Acidic Copper Sulphate Solutions," Surface Technology, Vol. 4, No. 3, 1976, pp. 227-235. doi:10.1016/0376-4583(76)90035-2

[7] L. Mirkova, N. Petkova, I. Popova and St. Rashkov, "The Effect of Some Surface Active Additives upon the Quality of Cathodic Copper Deposits During the Electrorefining Process," Hydrometallurgy, Vol. 36, No. 2, September 1994, pp. 201-213. doi:10.1016/0304-386X(94)90006-X

[8] H. M. A. Soliman, "Formalin Solution and Acetone as Organic Additives in Electrodeposition of Copper," Applied Surface Science, Vol. 195, No. 1-4, July 2002, pp. 155-165. doi:10.1016/S0169-4332(02)00553-6

[9] I. Z. Selim, K. M. El-Sobki, H. M. A. Soliman and A. A. Khedr, "Influence of Some Organic Solvents on Electrodeposition of Copper Using Vertical Parallel Plates," Bulletin of Electrochemical Industry, Vol. 16, No. 7, 2000, pp. 315-325.

[10] R. Winand, "Application of Polarisation Measurements in the Control of Metal Deposition," Elsevier Science, Amsterdam, 1984.

[11] D. R. Gabe and F. C. Walsh, "Enhanced Mass Transfer at 
the Rotating Cylinder Electrode. I. Characterization of a Smooth Cylinder and Roughness Development in Solutions of Constant Concentration," Journal of Applied Electrochemistry, Vol. 14, No. 5, 1984, pp. 555-564. doi:10.1007/BF00626299

[12] C. P. Wilde, S. V. De Cliff, K. C. Hui and D. J. L. Brett, "The Influence of Adsorbed Hydrogen and Recycling on the EQCM Response of Electrodeposited Pt Electrodes," Electrochim Acta, Vol. 45, No. 22-23, July 2000, pp. 3649-3658. doi:10.1016/S0013-4686(00)00448-5

[13] F. Franks, "Physicochemical Processes in Mixed Aqueous Solvents," American Elsevier, New York, 1967.

[14] M. Eisenberg, C. W. Tobias and C. R. Wilke, "Ionic Mass Transfer and Concentration Polarization at Rotating Electrode," Journal of Electrochemical Society, Vol. 101, No. 6, 1954, pp. 306-320. doi:10.1149/1.2781252

[15] A. A. Taha, "Study of the Effect of Ethylene Glycol and Glycerol on the Rate of Electropolishing of Copper by the Rotating Disk Technique," Anti-Corros Method M, Vol. 47, No. 2, 2000, pp. 94-104. doi:10.1108/00035590010316458

[16] I. M. Issa, M. M. Ghoneim, A. A. El-Samahy and M. Tharwat, "Polarographic Behaviour of Manganese (III) Complexes with Sulphate and Phosphate in Absence and Presence of Surfactants," Electrochim Acta, Vol. 17, No. 7, 1972, pp. 1251-1259. doi:10.1016/0013-4686(72)80012-4

[17] T. R. Beck and R. C. Alkire, "Occurrence of Salt Films during Initiation and Growth of Corrosion Pits," Journal of the Electrochemical Society, Vol. 126, No. 10, October 1979, pp. 1662-1666. doi:10.1149/1.2128772

[18] Y. N. Sadana and S. Nageswar, "Electrodeposition of Copper on Copper in the Presence of Dithiothreitol," Journal of Applied Electrochemistry, Vol. 14, No. 4, 1984, pp. 489-494. doi:10.1007/BF00610814

[19] I. Z. Selim, A. M. Ahmed and H. M. A. Soliman, "Use of The Rotating Cylinder Electrode to Study The Effect of Ethanol on Copper Electrodeposition," Bulletin of Electrochemical Industry, Vol. 17, No. 1, 2001, pp. 27-45.

[20] T. N. Andersen, C. H. Pitt and L. S. Livingston, "Nodulation of Electrodeposited Copper due to Suspended Particulate," Journal of Applied Electrochemistry, Vol. 13, No. 4, 1983, pp. 429-438. doi:10.1007/BF00617517

[21] G. A. Hope, G. M. Brown, D. P. Schweinsberg, K. Shimizu and K. Kobayashi, "Observations of Inclusions of Polymeric Additives in Copper Electrodeposits by Transmission Electron Microscopy," Journal of Applied Electrochemistry, Vol. 25, No. 9, 1995, pp. 890-894. doi:10.1007/BF00772211

[22] D. Russev, "An Electron Microscope Investigation of Electrolytic Copper Powders," Journal of Applied Electrochemistry, Vol. 11, No. 2, 1981, pp. 177-185. doi:10.1007/BF00610978

[23] D. Pletcher, I. Whyte, F. C. Walsh and J. P. Millington, "Reticulated Vitreous Carbon Cathodes for Metal Ion Removal from Process Streams: Part I: Mass Transport Studies," Journal of Applied Electrochemistry, Vol. 21, No. 8, 1991, pp. 659-666. doi:10.1007/BF01034042 